\title{
“MEASURING THE IMMEASURABLE"-THE EFFECTS OF TRADEMARK REGIMES: A CASE STUDY OF ARAB COUNTRIES
}

\begin{tabular}{|c|c|}
\hline \multicolumn{2}{|r|}{ Amir H. Khoury ${ }^{*}$} \\
\hline \multicolumn{2}{|r|}{ Table of Contents } \\
\hline Introduction & 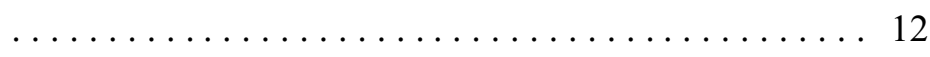 \\
\hline Chapter One & The Catalyst for This Research \\
\hline 1.1 & The Debate Surrounding Intellectual \\
\hline & Property Régimes . . . . . . . . . . . . \\
\hline 1.2 & The Gap in Conventional Literature \\
\hline 1.3 & Arab Countries and the Policy Debate. \\
\hline Chapter Two & A Model for Measuring Trademark Law in Action: \\
\hline & Present and Future Trademark Use $\ldots \ldots \ldots \ldots$. \\
\hline 2.1 & The "Absolute Level" \\
\hline 2.2 & The "Relative Level". \\
\hline 2.3 & The "Particular Level". \\
\hline 2.4 & The Concept of "Trademark Potential". \\
\hline & 2.4.1 Raw Materials \\
\hline & 2.4.2 Manufactured Products \\
\hline & 2.4 .3 Services $\ldots \ldots \ldots \ldots \ldots \ldots$ \\
\hline 2.5 & Assessing the "Trademark Potential" of \\
\hline & Arab Countries $\ldots \ldots \ldots \ldots \ldots \ldots$ \\
\hline & 2.5.1 Franchising $\ldots \ldots \ldots \ldots \ldots \ldots \ldots \ldots \ldots$ \\
\hline & 2.5.2 Services ..... \\
\hline & 2.5.3 Super Brands . \\
\hline Chapter Three & The "M.A.R.T.I.N.A." Scale . . . . . . . . . . . . \\
\hline 3.1 & Defining the M.A.R.T.I.N.A. Scale ... \\
\hline & 3.1 .1 Japan $\ldots \ldots \ldots \ldots \ldots \ldots \ldots \ldots$ \\
\hline & 3.1 .2 Israel $\ldots \ldots \ldots \ldots \ldots \ldots \ldots \ldots \ldots \ldots 6$ \\
\hline & 3.1 .3 Jordan $\ldots \ldots \ldots \ldots \ldots \ldots \ldots$ \\
\hline Chapter Four & Assessments and Conclusions $\ldots \ldots \ldots \ldots$ \\
\hline
\end{tabular}

* Dr. Amir H. Khoury, Lecturer, Tel Aviv University, Faculty of Law. 


\section{INTRODUCTION}

When the law sets out rules and standards it also sets in motion a complex set of mechanisms that determine how the law is implemented and how it functions. Thus, the "law in books" and the "law in action" are, in more ways than one, distinct creatures, separated at birth, leading separate lives. Trademark law is no exception.

This research introduces, through data analysis, a method for measuring and assessing the effects of trademark law on countries. In constructing this model I have focused on four Arab countries. However, the model itself is applicable to all countries. Indeed, the comparative data that is presented in this research relates to various countries around the world.

\section{Chapter One \\ The Catalyst for This Research}

The purpose of this research is to introduce a mechanism for measuring the effects of trademark laws by focusing on "the law in action" i.e., how the trademark régime is actually utilized.

I suppose that when first confronted with this topic, one might ponder the question as to why it is of any relevance; and more bluntly, why should the legal community be bothered with this seemingly trivial issue of examining the effects of trademark régimes? These skeptics might pose a question along these lines: would it not be wiser to focus our energy on the actual protection of rights in trademarks and not to attempt to examine the "effects" of trademark laws on a given economy?

This compelling question casts some doubt on the very merits of such an endeavor and might have even rendered it completely redundant, if it were not for the ongoing debate pertaining to intellectual property protection.

Indeed, this research has been prompted by three overlapping factors each of which, I believe, provides sufficient cause for conducting it.

\subsection{The Debate Surrounding Intellectual Property Régimes}

The global economy has created a "New World Order" with respect to intellectual property. The most potent characteristic of this order is its attempt to produce an ever-increasing unified system of protection for intellectual property rights. While these attempts have been largely successful thanks to decisive support by influential states and business interests, others have 
questioned their benefits for developing countries. Indeed, doubts about the justification for trademark protection are not new. Even in the United States, today's leading proponent of trademark protection, the advent of trademarks has not been without controversy. ${ }^{1}$

On the one hand, proponents of trademark protection contend that trademark protection is beneficial to the economies of developing countries in that it assists domestic producers to reap income that would otherwise be lost to counterfeiters. These proponents also argue that protecting foreign brands will encourage their owners to invest in developing countries and to establish an industrial or commercial presence therein thus opening their respective markets and creating new jobs therein.

Opponents to this approach contend that the above mentioned benefits of trademark protection do not apply in the case of developing countries because these countries are generally consumers rather than owners of trademarks. In addition, opponents contend that foreign investments do not contribute to the economies of developing countries because those investments are primarily "turnkey" projects that do not include a substantial transfer of technology or expertise.

In essence, the above mentioned debate merely constitutes a mini-cosmos of sorts for a much more expansive debate regarding the development of Third World countries. That debate is reflected in two opposing theories; namely, the "Development Theory" and the "Dependency Theory" which cover all spheres of development including economic structure and social values.

While the "Development Theory" urges developing countries to adopt modern norms of industrial countries, as a means for development, those citing the "Dependency Theory" contend that such adoption merely serves to widen the economic rift between the rich "North" and the poor "South." Literature is saturated with a variety of predictions regarding the effects of trademark protection. On one end of the scale there are those that view the adoption of intellectual property laws as a precondition for facilitating

1. The issue of protecting trademarks has collided repeatedly with concepts of national and international social justice. One unlikely source for such a debate took place during the legislation process of the United States trademark law- the Lanham Act. Initially the U.S. Department of Justice considered the Act as "too strongly favoring big business and allowing for illegal monopolies." See Carla Oakely, The United States Trademark Century in Review: Prosperity in the U.S. Leaps and Lulls, 1930 to 1959, available at http://www.inta.org/index.php?option=com_content\&task=view\&id=1291\&Itemid=126\& getcontent $=1$ (last visited May 15,2006) (noting that eventually the Lanham Act was accepted and was perceived "as a means to protect consumers from deception and essential for business to secure protection in the United States and abroad."). 
international trade and for securing development. ${ }^{2}$ Similarly, leading international organizations such as WTO, WIPO and INTA contend that standards of trademark protection (as formulated in TRIPS) are equally beneficial to developed countries and to developing countries. ${ }^{3}$

Further down the scale, other sources regard trademarks, and other forms of intellectual property rights (hereinafter "IPRs"), as non-universal norms not "applicable to non-industrialized societies." Others, at the far end of the scale, go even further by contending that IPRs are merely an economic tool of Western monopoly. ${ }^{5}$ Those further contend that modern standards of intellectual property (hereinafter "IP") protection are prone to nourish the monopoly that was created by developed countries and to widen the existing economic rift between developed and developing countries, this, due to the unequal distribution of IP across countries. Hence, they advocate for a new IP régime that is more receptive to the needs of developing countries. ${ }^{6}$

In earlier research, I have expansively explored both theories. For the purpose of this research suffice it to note that while the development theory sees the adoption of norms and standards as a key component in development, the dependency theorists contend that developing countries cannot develop by merely copying "Western" values and standards because they will always be dependent on "Western" capital and technology.

2. See Keith E. Maskus, Intellectual Property Rights in the Global Economy x (2000); Robert M. Sherwood, Human Creativity for Economic Development: Patents Propel Technology, 33 Akron L. Rev. 351 (2000); Robert J. Gutowski, Comment, The Marriage of Intellectual Property and International Trade in the TRIPS Agreement: Strange Bedfellows or a Match Made in Heaven?, 47 BufF. L. Rev. 713, 715 (1999).

3. See Peter Gallagher, Guide to the WTO and Developing Countries 16 (2000). See also MASKUS, supra note 2 (contending that the "balance of evidence strongly suggests" that IPRs provide an important foundation for promoting technology transfer, local innovation and economic growth in the long run and further asserting that in Lebanon and China trademark and trade secret infringement was at least as costly to domestic as to foreign firms, who had greater resources to deal with it and more options to withdraw from the market).

4. See Ruth L. Gana, The Myth of Development, the Progress of Rights: Human Rights to Intellectual Property and Development, 18 LAW \& POL'Y 315, 326 (1996).

5. See Martin Khor, How the South is Getting a Raw Deal at the WTO, in Views From the South: The Effects of Globalization and the WTO on Third World Countries 19, 22-23, 26-27 (Sarah Anderson ed., 2000). See also Walden Bello, Building an Iron Cage: The Bretton Woods Institutions, The WTO and the South, in Views From the South: THe EFfects of Globalization And THE WTO ON THIRd World Countries 74-77 (Sarah Anderson ed., 2000).

6. Peter Drahos, A Philosophy of Intellectual Property 204-05 (1996). See also Christopher May, a Global Political Economy of Intellectual Property Rights, The New ENCLOSURE? 155 (2000); Glynn S. Lunney, Trademark Monopolies, 48 EMory L.J. 367 (1999); MASKus, supra note 2 (contending that stronger protection of IPRs, by itself, is not enough to promote growth).

7. Amir H. Khoury, The Effects of Trademark Policy on Development: The Case of Arab 
Clearly, this ongoing debate does not, and cannot, provide a conclusive answer as per the effects of trademark laws on countries. That is because the bulk of existing literature is limited to theoretical arguments and does not provide data in order to substantiate anyone of these conflicting assertions. ${ }^{8}$

This research intends to fill this gap by introducing a model for measuring the effect of trademarks on developing countries, what is referred to as the "law in action" as opposed to the "law in books."

Examining every Arab, let alone every developing country, is not a viable option. Thus, and for the sake of simplicity, the proposed model focuses on four Arab countries that reflect the political-economic diversity among Arab countries and developing countries at large. These countries are: The Arab Republic of Egypt; The Kingdom of Saudi Arabia; The Hashemite Kingdom of Jordan and The Arab Republic of Syria. ${ }^{9}$ Notwithstanding this limitation,

Countries, in Intellectual Property, Trade and Development (Daniel Gervais ed., forthcoming Oct. 2007).

8. MASKus, supra note 2, at 156 (submitting that, on the one hand, "effective trademark enforcement both raises the average quality of products over time and provides a wider range of qualities from which consumers may choose." However, Maskus cautions that "[w]hile this statement is widely accepted by economists and business scholars and finds extensive anecdotal support, I have found no systematic econometric study of whether it applies in developing countries.").

9. Zvi. Y. Hershlag, Industrialization in Arab Countries: Patterns, Options and Strategies, in ARAB Industrialization and Economic Integration 13 (Robert Alibani ed., Croom Helm Ltd. 1979). Hershlag explains that: "Arab countries present a wide and diversified spectrum, both geographically and economically, apart from political, institutional and special aspects." It is worth noting that Arab countries are divided into three groups depending on their geographic location, namely; the Arab Peninsula, Levant Countries and North Africa. Except for Yemen, countries located in the Arab Peninsula are also widely referred to as the "Gulf States." These countries include Saudi Arabia, The United Arab Emirates (U.A.E), Kuwait, Oman, Qatar, Bahrain and they comprise the Gulf Cooperation Council (G.C.C.). The largest, wealthiest and most powerful member of this group is the Kingdom of Saudi Arabia. Hence, it has been selected to represent this group. The second group referred to as the "Levant Countries" includes Lebanon, Jordan, Iraq, Syria and the Palestinian Areas. Of this group, I focused on Jordan and Syria. The last group is comprised of countries located in North Africa. This group of Arab countries includes Egypt, Sudan, Tunisia, Algeria, Libya, and Morocco. Egypt is the most prominent member of this group and has the largest population. I have selected it to represent this group. Some regions of the Arab world are endowed with rich deposits of minerals namely Petroleum. Other Arab countries do not have such resources. Indeed, this distinction is held by some to be the most important one because it separates the "haves" from the "have-nots." Saudi Arabia for example represents the "haves" and Syria, to a large extent, represents the "have-nots." Egypt is not only the Arab country with the biggest population but is also among the most influential of Arab countries and the most dominant on the political scene. What is more, during the past three decades, Egypt has been striving to attain better ties with the West through economic openness. Furthermore, Egypt was the first Arab state to sign a formal peace treaty with Israel. Syria, on the other hand, has a relatively closed and underdeveloped economy. Jordan has a relatively small population and a weak but aspiring economy. It has strong ties with the United States and other Western countries and has signed a peace treaty with Israel. Saudi Arabia is the richest Arab country in the oil-rich Arabian Gulf. Much like Egypt, Saudi Arabia traditionally maintained firm connections with the West which have 
and in order to gain a better understanding, the research also considers other Arab countries.

According to the "Development Theory," developing countries are advised to "acquire modern cultural values and create modern economic and political institutions" that would act more like the West. ${ }^{10}$ This theory is based on the premise that the only way for non-industrialized countries to develop is by imitating developed countries and by adopting the latter's standards, values and conduct. ${ }^{11}$ In essence, this theory advocates a mechanical adoption and imitation of norms (including legal norms) undertaken by other countries in their strive towards attaining development and prosperity.

In this context, Development theorists suggest that "ICs have developed because of the existence of specific forms of laws and institutions, while nonICs failed to do so because they had no such laws and institutions or were "traditional." "12 In the context of trademark protection, the Development theory would typically suggest that developing countries that adopt modern standards of trademark protection would prompt their economies and industries to become developed.

On the other hand, the opposing "Dependency Theory" emanates from preceding economic theories by Carl Marx and Friedrich List. At its core, this theory submits that the world capitalist economy is a resultant of the domination by a few countries ("The Center") over most of Africa, Asia and Latin America ("The Periphery"). Dependency theorists contend that the "Center" has dominated the "Periphery" by employing various tactics and strategies including the formulation of an international division of labor (starting from the 16th century) in which countries of the "Periphery" were

\footnotetext{
experienced considerable strain in the wake of September 11th.

10. Howard Handelman, The Challenge of Third World Development 12 (1996).

11. Assafa Endeshaw, Intellectual Property Policy for Non-Industrial Countries, in LAW, SocIAL Change and Development Series 1996, at 11 (Dartmouth 1996); Zvi Yedhda Herschlag, The Philosophy of Development Revisited (Leiden 1984); Frank Cass, Development Theory: Four Critical Studies (David Lechmann ed., 1979); Francis G. Snyder, Law and Development in Light of the Dependency Theory, 14 LaW \& Soc'Y Rev. 723 (1980); Leonard J. Theberge, Law and Economic Development, 9 Denv. J. InT’L L. \& Pol’y 231 (1980); BuöRn Hettne, Development Theory And the Three Worlds: Towards an International Political Economy of Development (1990); Dependency Theory: A Critical Reassessment (Dudley Seers ed., 1981); Paulo Almeida, The Political Economy of Intellectual Property Protection: Technological Protectionism and Transfer of Revenue Among Nations, 10 Int'L J. Tech. Mgmt. 214 (1995); Handelman, supra note 10, at 14; Warner Baer, The Economics of Prebisch and ECLA, in Latin America: Problems in Economic Development (C.T. Nisbet ed., 1969).

12. Endeshaw, supra note 11 , at 5 .
} 
"condemned to agriculture." Thus, according to this line of reasoning, the "Periphery" was prevented from attaining industrialization and remained in the capacity of supplier of primary products. ${ }^{13}$

Dependency theorists further contend that this unbalanced exchange (between "Center" and "Periphery") is evident in the permanent transfer of value-added products from the "Center" to the "Periphery." That, in turn, is said to have caused a lack of foreign currency reserves and lack of capital accumulation in countries of the "Periphery." Thus, a "vicious circle" was created whereby countries of the "Periphery" were largely relegated to the production and export of food and raw materials and forced to trade for industrial imports on unfavorable terms. ${ }^{14}$ In the context of this research, dependency theorists would suggest that excessive protection of trademarks as well as other intellectual property rights only serves the industries of developed countries and will hamper the efforts of developing nations to compete in the global marketplace.

Dependency theorists call for severing these ties of dependency. In their view, the "Development" theory cannot justify stringent trademark protection in developing countries because the mere adoption of standards of trademark protection does not automatically entail economic benefits. ${ }^{15}$ Consequently, those opposing stringent IP protection have advanced alternative "Industrialization Strategies" (such as "Import Substitution") whereby developing countries can exercise self-sufficiency. ${ }^{16}$

13. Friedrich List, The National System of Political Economy (Samson S. Lloyd trans., Longmans, Green \& Co. 1885) (1841) (demonstrating that "free trade is inimical to domestic industrial development if undertaken with more foreign partners”); 1 KARL MARX, CAPITAL 1019-23 (Samuel Moore \& Edward Aveling trans., Foreign Languages Publishing House 1961) (1867). According to Endeshaw, this relationship has reflected negatively on the countries of the "Periphery" in various ways including the exchange process, the freedom of independent economic decision-making and the proliferation of technology. See Endeshaw, supra note 11, at 3.

14. See Handelman, supra note 10 , at 21 .

15. Id. at 29-30; see also Endeshaw, supra note 11, at 2-15 (expanding on the comparison between modernization and dependency). See also Maurice H. Dobb, Some Aspects of Economic Development (1951) (noting that the problem of industrialization is, in essence, not of finance, but of economic organization; research submits that economic independence is not attainable unless governments undertake radical economic and social changes which encompass a wide array of issues including national economy, poverty, illiteracy, as well as, imperialistic monopolization).

16. Dependency theorists submit that developing countries cannot develop by merely copying "Western" values and standards because, in this way, they will become dependent on "Western" capital and technology. Therefore, these theorists advocate a proactive approach by developing countries mainly through self-sufficiency and ultimately by competing for international export markets. See also HANDELMAN, supra note 10, at 226 (discussing the various "Industrial Strategies" undertaken by some third world countries, including ISI-ISI-Import Substituting Industrialization (e.g. in Latin America) and EOIExport-Oriented Industrialization (e.g. the "Asian Tiger Economies")). 


\subsection{The Gap in Conventional Literature}

Literature pertaining to the effects of trademark laws on developing countries approaches the topic from a legalistic and formalistic angle; namely by highlighting basic moral concepts calling for fair competition and fair dealing in trade and by identifying the amendments and measures that are necessary in order to comply with internationally accepted standards of trademark protection. Thus, the bulk of literature is saturated with formalistic materials pertaining to the perceived benefits of trademark protection without presenting data to that effect.

Contrary to this type of literature, other literature attempts to examine the "law in action" i.e. the actual use of trademarks. ${ }^{17}$ In this regard, one commentary contends that before turning to study trademark laws, it is first necessary to clarify "the role played by trademarks in developing countries in trade and in connection with the process of industrialization." "18 That type of research also considers the relationship between protection for IPRs and economic development. ${ }^{19}$ Notably, in its research, the United Nations Conference on Trade and Development (UNCTAD) has examined (empirically) the relationship between the number of trademark registrations originating in a given country and that country's economy and export market. ${ }^{20}$

17. See Douglas F. Greer, The Economic Benefits and Costs of Trademarks: Lessons for Developing Countries, 7 World Dev. 683 (1979); Peter O'Brien, Trademarks in Developing Countries, 14 J. Modern Afr. Stud. 297 (1976); Surendra J. Patel, Editor's Introduction, 7 World Dev. 649 (1979); Surendra J. Patel, Trademarks and the Third World, 7 World Dev. 653 (1979); Richard T. Rapp \& Richard P. Rozek, Benefits and Costs of Intellectual Property Protection in Developing Countries, 5 J. World TRAde 75 (1990).

18. Sándor Vida, Trade Marks in Developing Countries 22 (1981).

19. MASKuS, supra note 2, at 10-11, 87-197 (specifically considering Patent Applications; Trademark Applications; Applications for Registrations of Plant Varieties; Scope of demand for copyrighted Products; Trade in IPR-Sensitive Goods for Selected Countries; Trade in IPR-Sensitive Services and Royalties and License Fees; Inward and Outward Stocks of Foreign Direct Investment; Membership Trends in Key Intellectual Property Conventions; Indications of the Strength of IPRs Laws; Estimated Rates of Software Piracy and Lost Revenues; Simulated Increases in Total Imports by Sector into Developing Countries Resulting from Strengthened Patent Laws; Percentage of Firms Claiming that Strength or Weakness of IPRs has a Strong Effect on the Level of Direct Investment; Estimators of How TRIPS Patent Changes Affect International Flows of Economic Activity for Selected Countries); see also United Nations Conference on Trade and Development (UNCTAD), The Impact of Trademarks on the Development Process of Developing Countries, U.N. Doc. TD/B/C. 6/AC.3/3, Geneva, Switz. (June 1977).

20. United Nations Conference on Trade and Development (UNCTAD), Systems Including Industrial Property Systems for Improving the National Scientific and Technological Infrastructure of Developing Countries, U.N. Doc. TD/B/C.G/AC.2/4 Geneva, Switz. (1975). 
Additional research dealing with the economic effects and implications of intellectual property protection has factored a host of empirical date into a single model, the aggregate of which, provides an indication as to the effects of legal norms. For example, a recent research has investigated the economic effects of patent protection by analyzing data pertaining to a host of factors including: rating innovative technical fields; the ratio of non-resident/resident patent applications in all industries (referred to, by that research, as the "dependency ratio"); number of patent applications; number of patent applications for a country and the ratio of resident and non-resident patent applications for patents. ${ }^{21}$ Another research that is even more recent examined the link between intellectual property rights and economic growth by analyzing data pertaining to: export market share in technology intensive products (for the years 1970, 1980 and 1990); research and development expenditure as a percentage GNP; non-defense research expenditures as a percent of GNP; corporate profits (1959-1995); merchandise imports and exports; number of drugs introduced in the U.S. (1940-1979); foreign sales of U.S. software (1986-1991); estimates of market share for pirated video cassettes; estimated U.S. trade losses (1993); gross revenues of selected films and net foreign direct investment in China (1980-1994). ${ }^{22}$

A third research that assesses the economic effects of IP régimes analyses various relevant data pertaining to: patent applications in selected countries; trademark application in selected countries; applications for registrations of plant varieties in selected countries; indications of demand for copyright products in selected countries; trade in IPR-sensitive goods for selected countries; trade in IPR-sensitive services and royalties in license fees; membership trends in key IP conventions; qualitative trends in IP protection in selected countries and estimated rates of software piracy and lost revenue. ${ }^{23}$ Another research did not only consider the number of patents registered in a certain country, but also assessed the degree of "innovation" of those patents by examining how frequently they had been cited in other patent claims. ${ }^{24}$

21. Nikolaus Thumm, Intellectual Property Rights: National Systems and HARMONIZATION IN EUROPE (2000). This research also includes a survey in the form of a questionnaire that was sent to various biotechnological firms throughout Europe. Id. at 80-94, 137-39.

22. Robert L. Ostergard, Jr., The Development Dilemma: The Political Economy of Intellectual Property Rights In the International System 83-95, 102-06, 128 (2003).

23. MASKUS, supra note 2, at 68, 70-77, 80-92, 100.

24. Lee Branstetter, Measuring the Impact of Academic Science on Industrial Innovation: The Case of California's Research Universities (May 15, 2007), http://www.nber.org/CRIW/papers/branstetter.pdf. 
Evidently, the bulk of research dealing with the effects of IP protection focuses on patents. ${ }^{25}$ Far less research has been devoted to other types of IP including copyrights, trade secrets and trademarks. ${ }^{26}$ Furthermore, the bulk of research attempting to tackle the quandary pertaining to the effects of intellectual property régimes does so by presenting theoretical assertions and, in some cases, by providing anecdotal support. Indeed, most literature that supports trademark protection refers to the basic attributes (and functions) of trademarking but refrains from crossing beyond that threshold into the domain in which the rationales of trademarking are not taken for granted but, rather, are scrutinized vis-à-vis empirical data. ${ }^{27}$ Indeed, most of the literature dealing with the effects of intellectual property protection in developing countries is limited to theoretical arguments with little or no empirical support. In this regard, one recent study observed that "though there are extensive treaties on the subject by legal scholars and international relations specialists, they tend to take economic ramifications for granted rather than

25. For one example of IP related research in Arab countries that focuses primarily on patents rather than trademarks, see KenA'An Al-Ahmar, The Role of IP AND Indu strial Property ANd E-commerce IN GROWTH (2000). Al-Ahmar contends that the protection of inventions and creations is a precondition for maintaining inventiveness. Also, the protection of IP and industrial property is a condition for the organization of competition and for a healthy performance of the economy. Furthermore, such protection serves the interests of consumers. In this regard Al-Ahmar focuses on patent protection, incentives to produce, transfer of technology, allowing for the development of similar inventions. Patents and trademarks have different rationales. While patents center on $R \& D$ and inventiveness, trademarks operate primarily as a marketing tool. Thus, empirical research that reflects on patent protection cannot be unreservedly applied to trademarks.

26. Research generally associates IP protection with patents. See Robert M. SHerwood, Intellectual Property and Economic Development 67-82 (1990); see also Michael R. Gadbaw \& Timothy J. Richards, Intellectual Property Rights, Global Consensus, Global Conflict? (Boulder, Westview Press 1988); Carlos Alberto Primo Braga, Intellectual Property Rights and the GATT: A View from the South, 22 Vand. J. Transnat'L L. 243 (1989); Friedrich-Karl Beier, The Significance of the Patent System for Technical, Economic and Social Progress, 5 IIC InT'L Rev. Indus. Prop. \& Copyright L. 563 (1980); Siegfried Greif, The Role of Patent Protected Imports in the Transfer of Technology to Developing Countries, 10 IIC Int'L Rev. Indus. Prop. \& Copyright L. 124 (1979); Siegfried Greif, Patents and Economic Growth, 18 IIC InT'L Rev. Indus. Prop. \& Copyright L. 191 (1987); Hans Peter Kunz-Hallstein, The Revision of the International System of Patent Protection in the Interest of Developing Countries, 10 IIC INT'L Rev. Indus. Prop. \& COPYRIGHT L. 650 (1979); Barbara Hansen, Economic Aspects of Technology Transfer to Developing Countries, 11 IIC INT'L REv. Indus. Prop. \& Copyright L. $430-40$ (1980); Hanns Ullrich, The Importance of Industrial Property Law and Other Legal Measures in the Promotion of Technological Innovation, 1989 INDUS. PROP. 102.

27. For a detailed survey of this type of literature, see Khoury, supra note 7, at 15; EDWAR EID, Business and Traders and Commercial Institutions 463-65 (1971); Samiha Al-Kiloobi, Commercial Law 254 (1999); Samiha Al-Kiloobi, Industrial and Commercial Property 240 (1968). 
analyze their subtleties and test them with empirical evidence." ${ }^{28}$ In this regard, one research has observed that there is no systematic econometric study of whether that anecdotal support applies in developing countries. ${ }^{29}$ Another commentary observed that literature "did not have in mind the detailed interests of developing countries," and did not analyze the particular problems of these countries, nor reflect on their specific problems with respect to trademark legislation. ${ }^{30}$ In this regard, that commentary observes that trademarks "play a variety of roles, which differ from industry to industry and which may produce different balances of advantages and disadvantages." ${ }^{\prime 1}$ Consequently, some commentators recommend conducting a thorough review of the TRIPS' provisions in order to achieve a balance between IPRs and the national interests of developing countries. ${ }^{32}$

Thus, my research constitutes an integral part of the general discourse relating to the link between intellectual property and development. Indeed, my research aims to contribute towards filling the gap that exists in literature namely by examining the effects of trademark protection on developing countries through data analysis.

28. Maskus, supra note 2, at 7. See also Sherwood, infra note 30, at 1 (noting that "the literature is replete with studies of the relation of innovation to economic growth, but the relation between intellectual property protection and innovation in developing countries rests largely on conjecture."); MASKUS, supra note 2, at 2 (noting that "[a]lthough patents have been studied in various ways for their role in developed country economies, literature relevant to developing countries is scant.").

29. MASKUS, supra note 2, at 156.

30. VIDA, supra note 18, at 21. The literature dealing with the effects of trademark protection in developing countries typically focuses on regions other than the Middle East (i.e. the Far East, Latin America, the Indian Sub-Continent and Africa). Also see Endeshaw, supra note 11, at 12; Robert M. Sherwood, Intellectual Property Systems and Investment Stimulation: The Rating of Systems in Eighteen Developing Countries, 37 IDEA 261 (1997).

31. W.R. Cornish \& Jennifer Phillips, The Economic Functions of Trade Marks: An Anal ysis with Special Reference to Developing Countries 41 (IIC 01/1982). See also Economic Council of Canada, Report on Intellectual and Industrial Property 181-219 (1971); O’Brien, supra note 17, at 297-309; Guido Di Tella, The Manipulation of Demand: The Problem of Trade Marks, 1 World Dev. 35-42 (1971); Mayer Gabay, The Role of Trademarks and Development in Developing Countries, 20 Indus. Prop. 102 (1981); United Nations Conference on Trade and Development (UNCTAD), Geneva, Switz., The Impact of Trademarks on the Development Process of Developing Countries U.N. Doc. TD/B/C. 6/AC.3/3 (June 1977).

32. Fattoh Abed El-Galil Hamed, Working Paper on IP and the Expected Changes in Light of GATT 3-5, The Egyptian Academy of Scientific and Technological Research. As such, that commentary does not accept the TRIPS face value but rather recommends following a balanced approach; by identifying the virtues as well as the pitfalls of that agreement. Thus, this approach attempts to boost national research and technology while not taking too radical an approach that may hamper foreign investments. 


\subsection{Arab Countries and the Policy Debate}

In order to extenuate the research question, it is imperative to shed some light on the trademark laws in the four Arab countries that constitute the subjects of the case study in this research and to examine the compatibility of these laws with international standards of trademark protection.

In view of their ever-increasing economic value, trademarks, much like other types of intellectual property rights (IPRs), received protection within a wide array of international as well as regional agreements and national laws. The Paris Convention for the Protection of Industrial Property in 1883 was the first multilateral international agreement intended to protect industrial property including trademarks. Since then, other agreements and treaties have been introduced culminating in the Agreement on Trade Related-Aspects of Intellectual Property Rights (TRIPS).$^{33}$

TRIPS regulates various issues relating to IPRs including availability; scope; use; enforcement and multilateral dispute settlement procedures. TRIPS requires member states to establish a minimum level of intellectual property protection in their respective national law. With respect to trademarks, TRIPS sets various standards, including: recognition of service marks; setting a minimum (renewable) term of protection; defining use requirements; enhancing the role of customs in enforcement and recognizing well-known marks even if not registered in the specific jurisdiction. ${ }^{34}$ In addition, TRIPS allows for canceling the registration of a mark due to non-use (subject to exceptions); confirming the right to use a mark without conditions; and regulating issues of licensing and assignment.

The TRIPS obligations, undertaken by members of the WTO, are rigorously implemented. ${ }^{35}$ TRIPS sanctioned the lack of IP protection by subjecting non-complying countries to economic and trade sanctions imposed through the WTO Dispute Resolution Mechanism or by expelling such a member state from that organization. ${ }^{36}$ Thus, TRIPS revolutionized the rules

33. TRIPS came into effect on January 1, 1995. See generally Marshall A. Leaffer, The New World of Intellectual Property Law, 2 MArq. IntelL. Prop. L. Rev. 1 (1998). For a concise summary of TRIPS, see Main Provisions of the TRIPS Agreement, available at $\mathrm{http} / /$ www.southcentre.org/publications/trips/ toc.htm (last visited May 15, 2007); see also Daniel Gervais, The TRIPS Agreement: Drafting History AND ANAL Ysis (Sweet \& Maxwell 1998).

34. MASKUS, supra note 2, at 15-26.

35. GALlAGHER, supra note 3 , at 53,181. The deadline for implementation of TRIPS into national law depends on how that country is classified; namely: "Developed Countries" (until 1995); "Developing Countries" (until January 2000); "Least-Developed Countries" (until 2006).

36. TRIPS allows for settling IP related disputes between member states by applying the WTO 
of IP protection because of its substantive rules, its ability to harmonize the legal régimes and its ability to ensure the adoption and continued enforcement of these norms. ${ }^{37}$ Consequently, it is not surprising that TRIPS is widely regarded as the most central agreement in the context of IP protection. ${ }^{38}$ Significantly, the legal norms pertaining to intellectual property rights have ceased to be shaped in accordance with national norms and interests, and have become intertwined in the wider context of international trade. ${ }^{39}$ In fact, by including the IP régime of TRIPS within the WTO, the lack of protection for IPRs is now viewed as an impediment to trade, much like dumping and subsidies. ${ }^{40}$

Today, the overwhelming majority of Arab countries are members to all of the main treaties, agreements and conventions pertaining to intellectual property protection, and primarily the Paris Convention (16 Arab countries) and the TRIPS agreement (1994) (11 Arab countries). Furthermore, as my previous expansive research indicates, all of these countries have enacted new intellectual property laws. ${ }^{41}$ Indeed, Arab countries have actively and diligently adopted, into their national legislation, modern standards of

"Dispute SettlementMechanism." This mechanism also covers disputes pertaining to "National Treatment" and "Most Favored Nation" status. Other issues such as Gray Market (parallel imports) have been excluded from TRIPS and the dispute settlement mechanism. See Gallagher, supra note 3, at 182.

37. May, supra note 6, at 76; David W. Plant, Resolving International Intellectual Property Disputes (International Chamber of Commerce Publishing 1999). Notwithstanding these uniform standards, TRIPS allows its members to exercise some independence, including: the right to limit the scope of rights within certain bounds, the right to grant compulsory licenses under certain conditions, and to take measures to prevent abusive anti-competitive practices. GALLAGHER, supra note 3, at 181 .

38. World Intellectual Property Organization, Introduction to Intellectual Property: Theory and Practice 475 (Kluwer Law International 1997); see also International Trademark Association (INTA), TRIPS 2000 Subcommittee Treaty Analysis Committee, Developing COUntries Compliance with the TRIPS AgREEMENT, available at $\mathrm{http} / /$ www.inta.org/downloads/tap trips2000.pdf (last visited May 15, 2007).

39. John H. Jackson et al., Legal Problems of International Economic Relations 291, 885-92 (3d ed. 1995).

40. The Ministerial Declaration on the Uruguay Round, stated that

in order to reduce the distortions and impediments to international trade, and taking into account the need to promote effective and adequate protection of intellectual property rights and to ensure that measures and procedures to enforce intellectual property rights do not themselves become barriers to legitimate trade, the negotiations shall aim to clarify GATT provisions and elaborate on appropriate new rules and disciplines.

http://www.sice.oas.org/trade/Punta_e.asp (last visited Aug. 3, 2007); SHERwood, supra note 26, at 67-92; see also Endeshaw, supra note 11, at 121; MAY, supra note 6, at 78.

41. For more details and expansive research on this matter, see Amir H. Khoury, The Development of Modern Trademark Legislation and Protection in Arab Countries of the Middle East, 3 J.L. \& TeCH. 233 (2003), and Amir H. Khoury, Trademark Policy: The Case of Arab Countries, in Intellectual Property, Trade and Development (Daniel Gervais ed., forthcoming Oct. 2007). 
intellectual property protection as prescribed by various international agreements and mainly TRIPS and have been expending efforts with respect to trademark protection and enforcement. ${ }^{42}$ In so doing, Arab countries appear to have adopted the approach advocated by Development theorists; specifically, that trademark protection will yield positive effects. Thus, the underlying assumption of my research is that Arab countries are operating within a clear, effective and modern trademark régime.

Considering the three above mentioned factors, the question persists: How, if at all, would it be possible to measure the effects of trademark régimes in countries and to assess the "law in action" therein.

\section{Chapter Two \\ A Model for Measuring Trademark Law in Action: Present and Future Trademark Use}

Thus far, we have alluded to the fact that, in literature, there are conflicting views regarding the ramifications of trademark régimes. On the one hand stands the assertion that trademarks are essential for creating a viable market and for stimulating and attaining economic growth. On the other hand is the contention that in developing countries, the trademark system merely benefits foreign brand owners. "In attempting to provide some objective mechanism for measuring the effects of trademark régimes, I propose a model containing two levels namely; the 'trademark balance' and the "trademark potential." The "Trademark Balance" comprises three elements, namely the:

a. "Absolute" number of registrations and/or applications in a given country;

b.. "Relative" trademark registration by residents and non-residents;

c. "Particular" countries that dominate trademark registration in a given country.

Examining the scope of trademark registration in a country on these three levels can assist in identifying trends in trademark use, ownership and utilization. $^{43}$

42. Jordan, Egypt and Lebanon have already undertaken such commitments of comprehensively amending their IP laws. Moreover, substantial legal literature within Arab countries advocates the adoption of modern standards of IP protection, including trademarks, as a pre-condition for transforming these countries into modern economies. See A'Amer Al-Ksiwani, Intellectual Property 12, 58 (1998); Sayed Hassan Amin, Intellec tual Property and Industrial Property in the Middle East (1992).

43. The data pertaining to trademark registration originates in a variety of sources including: World 
The data pertaining to trademark registration has important economic significance because trademark law accords protection to trademarks based on their registration. Marks that are not registered generally do not benefit from the trademark régime. ${ }^{44}$ Consequently, data pertaining to trademark registration in a specific jurisdiction and the identity of their owners is indicative of the brands that are being used and protected by law in the relevant jurisdiction. In addition, the absolute number of trademark registrations is indicative of the number of products and services that are on sale in the market. This in turn reflects the economic vitality of that market.

The "Trademark Potential" of a country is based on its trade structure. This is determined by considering empirical data pertaining to imports and exports as well as the type of production that dominates Arab economies. This data helps to predict the degree of future trademark use in Arab countries, i.e. their "Trademark Potential."

In essence, the "Trademark Potential" is the aggregate between the types of products and services that are produced in a certain country, and the ability to sell these products and services under trademarks and service marks. For example, if a country produces raw materials that are shipped in bulk before processing, then it is possible to conclude that the country has a very low trademark potential.

In this regard, special attention is accorded to the franchising and service sectors because they both involve substantial use of marks. In addition, some emphasis is placed on the relationship between Arab corporations and leading brands. Furthermore, special attention is devoted to the petroleum industry because of its dominant share in the GDP and exports of Arab countries.

Thus, the combination of both factors (i.e. the "Trademark Balance" and the "Trademark Potential") reflects the use of trademarks within Arab countries both at present and in the future and shows how the trademark régime is being utilized by local and foreign brand-owners therein.

Intellectual Property Organization (WIPO); the United Nations Conference on Trade and Development (UNCTAD); the International Trademark Association (INTA); the World Trade Organization (WTO); the U.S. Trade Representative (USTR); and Arab Government agencies. See, e.g., The Office of the United States Trade Representative (USTR), 2001 Trade Policy Agenda and 2000 Annual Report, available at http://www.ustr.gov/Document_Library/Reports_Publications/2001/2001_Trade_Policy_Agenda/Section_ Index.html (last visited May 15, 2007) (indicating that the Far East and the Middle East remain hotspots for counterfeit toiletries, foods as well as luxury goods).

44. The only exception being well-known marks that are protected (in a limited manner) despite not being registered in the jurisdiction. 


\subsection{The "Absolute Level"}

The "Absolute" level considers the overall volume of trademark "traffic" in a given country in terms of the number of trademark registrations therein. In order to provide some comparative insight with respect to the number of trademark registrations, the data pertaining to Arab countries is contrasted with those of other countries around the world. The next three tables depict the scope of trademark registration in various countries for the period (1973-2000). Each of these tables focuses on a different segment of that period, namely; 1973-1982, 1984-1991 and 1992-2000. ${ }^{45}$

Table 1

The Absolute Level

Trademark Registrations - In Selected Countries

(1973-1982)

\begin{tabular}{|c|c|c|c|c|c|c|c|c|c|c|}
\hline COUNTRY & 1973 & 1974 & 1975 & 1976 & 1977 & 1978 & 1979 & 1980 & 1981 & 1982 \\
\hline Egypt & 587 & 536 & 656 & 531 & 704 & 632 & 554 & 615 & $\mathrm{n} / \mathrm{a}$ & 539 \\
\hline Syria & 796 & 674 & 820 & 800 & 800 & 949 & 625 & 1,200 & 709 & 762 \\
\hline Jordan & 683 & 93 & 396 & 588 & 920 & 658 & 792 & 1,103 & 700 & 473 \\
\hline Algeria & 783 & 1,140 & 503 & 682 & 229 & 1,425 & 580 & 681 & 772 & 2,207 \\
\hline Israel & 1,478 & 1,176 & 1,328 & 1,038 & 1,158 & 1,164 & 1,077 & 1,185 & 961 & 1,080 \\
\hline Turkey & 2,127 & 2,070 & 1,728 & 1,532 & 1,957 & 2,997 & 2,310 & 3,295 & 1,941 & 2,294 \\
\hline United States & 26,112 & 27,381 & 30,931 & 26,326 & 25,858 & 29,630 & 20,485 & 18,885 & 42,702 & 42,444 \\
\hline United Kingdom & 11,266 & 10,626 & 11,440 & 12,195 & 10,093 & 10,643 & 10,036 & 6,708 & 9,542 & 13,134 \\
\hline France & 23,736 & 23,723 & 16,957 & 15,618 & 45,167 & 35,745 & 51,177 & 47,116 & 32,330 & 35,452 \\
\hline Germany* & 12,251 & 12,808 & 12,828 & 12,718 & 13,350 & 13,855 & 14,217 & 16,844 & 14,699 & 15,086 \\
\hline Japan & 71,888 & 90,502 & 109,166 & 95,945 & 113,162 & 88,852 & 52,291 & 67,539 & 68,314 & 86,125 \\
\hline Spain & 18,134 & 13,975 & 14,662 & 23,206 & 32,645 & 38,354 & 45,346 & 18,152 & 16,766 & 19,375 \\
\hline India & 3,558 & 3,303 & 3,659 & 4,756 & 4,204 & 4,025 & 3,352 & 3,100 & 3,090 & 3,230 \\
\hline South Korea & 7,008 & 5,955 & 2,950 & 4,419 & 3,989 & 7,504 & 7,006 & 7,845 & 6,769 & 7,793 \\
\hline Argentina & 18,025 & 14,101 & 14,460 & 19,249 & 20,339 & 19,587 & 19,031 & 19,300 & 12,371 & 12,231 \\
\hline
\end{tabular}

*Federal Republic of Germany

45. For the first period, see World Intellectual Property Organization, Intellectual Property Statistics, WIPO, Geneva, 1984. For the period 1984-2000, see WIPO, http://www.wipo.int (last visited May 28, 2006). The data provided by WIPO is presented on a country by country basis. The tables that I have constructed in this chapter are a compilation of data. Some countries have not always provided WIPO with data. In those cases the slot has been marked with "n/a" (not available). 
Table 2

The Absolute Level

Trademark Registrations-In Selected Countries (1984-1991)*

\begin{tabular}{|c|c|c|c|c|c|c|c|c|}
\hline COUNTRY & 1984 & 1985 & 1986 & 1987 & 1988 & 1989 & 1990 & 1991 \\
\hline Egypt & 654 & $n / \mathrm{a}$ & 694 & $n / \mathrm{a}$ & 743 & $n / a$ & n/a & 894 \\
\hline Jordan & $\mathrm{n} / \mathrm{a}$ & $\mathrm{n} / \mathrm{a}$ & $\mathrm{n} / \mathrm{a}$ & $\mathrm{n} / \mathrm{a}$ & $\mathrm{n} / \mathrm{a}$ & 995 & $\mathrm{n} / \mathrm{a}$ & $\mathrm{n} / \mathrm{a}$ \\
\hline Saudi Arabia & $\mathrm{n} / \mathrm{a}$ & 1,756 & $\mathrm{n} / \mathrm{a}$ & $\mathrm{n} / \mathrm{a}$ & 1,825 & 2,063 & $\mathrm{n} / \mathrm{a}$ & 2,241 \\
\hline Morocco & 1,296 & 1,645 & 1,331 & 1,906 & 1,986 & 1,805 & 1,909 & 2,067 \\
\hline Algeria & 2,292 & 1,100 & 1,898 & 3,773 & 3,904 & 4,280 & 4,294 & 4,206 \\
\hline Tunisia & 480 & 553 & 725 & $\mathrm{n} / \mathrm{a}$ & $\mathrm{n} / \mathrm{a}$ & 1,374 & 1,431 & 1,565 \\
\hline Bahrain & 860 & 628 & 607 & $\mathrm{n} / \mathrm{a}$ & $\mathrm{n} / \mathrm{a}$ & $\mathrm{n} / \mathrm{a}$ & $\mathrm{n} / \mathrm{a}$ & $\mathrm{n} / \mathrm{a}$ \\
\hline Israel & 986 & 667 & 1,384 & 927 & 1,426 & 1,260 & 1,550 & 2,122 \\
\hline Turkey & 3,138 & $\mathrm{n} / \mathrm{a}$ & 4,289 & 4,557 & 4,873 & 5,084 & $\mathrm{n} / \mathrm{a}$ & 7,112 \\
\hline United States & 55,499 & 54,486 & 52,648 & 53,533 & 53,229 & 62,483 & 61,343 & 46,647 \\
\hline United Kingdom & 16,083 & 16,154 & 17,089 & 14,401 & 14,381 & 22,374 & 28,389 & 30,421 \\
\hline France & 38,588 & 37,192 & 58,151 & 64,201 & 70,581 & 74,719 & 79,052 & 90,549 \\
\hline Germany & 14,258 & 13,515 & 17,731 & 18,687 & 20,188 & 23,897 & 22,976 & 26,471 \\
\hline Japan & 116,636 & 119,534 & 118,475 & 117,075 & 119,287 & 119,598 & 117,185 & 95,329 \\
\hline Spain & 11,063 & 14,697 & 16,934 & 22,893 & 22,114 & 23,663 & 36,020 & 45,091 \\
\hline India & 2,240 & 1,870 & 2,965 & 3,075 & 4,366 & 5,335 & 6,429 & 7,425 \\
\hline Brazil & 17,699 & 21,571 & 17,057 & 21,340 & 33,198 & $\mathrm{n} / \mathrm{a}$ & 34,314 & 22,333 \\
\hline Mexico & 7,822 & 6,968 & 7,614 & 18,824 & 17,331 & 13,792 & 17,538 & 14,237 \\
\hline Greece & 2,834 & 3,814 & $\mathrm{n} / \mathrm{a}$ & 5,632 & $\mathrm{n} / \mathrm{a}$ & $\mathrm{n} / \mathrm{a}$ & $\mathrm{n} / \mathrm{a}$ & $\mathrm{n} / \mathrm{a}$ \\
\hline Canada & 12,205 & 11,236 & 12,323 & 13,718 & 13,678 & 14,362 & 13,758 & 14,597 \\
\hline Argentina & 24,158 & $\mathrm{n} / \mathrm{a}$ & $\mathrm{n} / \mathrm{a}$ & $\mathrm{n} / \mathrm{a}$ & 62,849 & $\mathrm{n} / \mathrm{a}$ & $\mathrm{n} / \mathrm{a}$ & $\mathrm{n} / \mathrm{a}$ \\
\hline
\end{tabular}

*No data is provided by WIPO for the year 1983.

Table 3

The Absolute Level

Trademark Registrations-In Selected Countries (1992-2000)

\begin{tabular}{|c|c|c|c|c|c|c|c|c|c|}
\hline COUNTRY & 1992 & 1993 & 1994 & 1995 & 1996 & 1997 & 1998 & 1999 & 2000 \\
\hline Egypt & $\mathrm{n} / \mathrm{a}$ & $\mathrm{n} / \mathrm{a}$ & $\mathrm{n} / \mathrm{a}$ & $\mathrm{n} / \mathrm{a}$ & 2,285 & 2,630 & 2,841 & 2,690 & 3,155 \\
\hline Syria & $\mathrm{n} / \mathrm{a}$ & $\mathrm{n} / \mathrm{a}$ & $\mathrm{n} / \mathrm{a}$ & 2,849 & 2,640 & $\mathrm{n} / \mathrm{a}$ & 3,306 & $n / \mathrm{a}$ & 4,297 \\
\hline Jordan & $\mathrm{n} / \mathrm{a}$ & $n / a$ & 2,138 & 2,462 & 4,000 & 4,085 & 2,352 & 2,494 & 2,536 \\
\hline Morocco & 2,348 & 4,220 & 4,702 & 4,564 & 9,654 & 3,312 & 6,908 & 3,283 & 3,579 \\
\hline Algeria & 5,115 & 5,799 & 5,240 & 4,848 & 4,242 & 2,362 & 3,702 & 3,912 & 4,565 \\
\hline Tunisia & $n / \mathrm{a}$ & 4,593 & 2,354 & 2,719 & 2,884 & $n / \mathbf{a}$ & $n / \mathbf{a}$ & $\Pi / \mathrm{a}$ & $n / \mathbf{a}$ \\
\hline Bahrain & 738 & $n / \mathbf{a}$ & $n / \mathrm{a}$ & $\mathrm{n} / \mathrm{a}$ & $n / \mathrm{a}$ & $n / \mathrm{a}$ & $n / \mathrm{a}$ & $\mathrm{n} / \mathrm{a}$ & $n / \mathrm{a}$ \\
\hline Israel & 2,154 & 3,371 & 4,577 & 5,637 & 5,782 & 7,023 & 7,217 & 6,995 & 7,425 \\
\hline Turkey & 7,185 & 7,837 & 9,346 & 5,445 & 7,202 & 11,321 & 7,464 & 20,401 & 20,875 \\
\hline United States & 80,173 & 80,614 & 63,903 & 85,557 & 93,714 & 138,155 & 129,871 & 87,431 & 109,544 \\
\hline United Kingdom & 35,137 & 32,934 & 28,828 & 33,400 & 57,470 & 49,035 & 48,600 & 51,441 & 65,649 \\
\hline France & 89,023 & 92,232 & 78,774 & 60,712 & $\mathrm{n} / \mathrm{a}$ & $\mathrm{n} / \mathrm{a}$ & $\mathrm{n} / \mathrm{a}$ & $\mathrm{n} / \mathrm{a}$ & $\mathrm{n} / \mathrm{a}$ \\
\hline Germany (Fed) & 29,283 & 37,080 & 45,207 & 21,934 & 40,256 & 57,435 & 57,919 & 61,039 & 70,279 \\
\hline Japan & 156,040 & 158,685 & 147,191 & 144,911 & 178,251 & 253,272 & 132,066 & 123,656 & 97,110 \\
\hline Spain & 84,216 & 59,326 & 63,669 & 69,642 & 81,017 & 59,374 & 59,810 & 63,086 & 73,919 \\
\hline India & 5,990 & 5,202 & 5,646 & 5,310 & 4,436 & $\mathrm{n} / \mathrm{a}$ & 4,840 & 8,010 & $n / a$ \\
\hline Brazil & 37,964 & 28,681 & 22,794 & 25,330 & 31,202 & $\mathrm{n} / \mathrm{a}$ & $\mathrm{n} / \mathrm{a}$ & $\mathrm{n} / \mathrm{a}$ & $\mathrm{n} / \mathrm{a}$ \\
\hline Mexico & 24,820 & 20,441 & 33,717 & 29,954 & 25,756 & 27,821 & 28,362 & 40,314 & $\mathrm{n} / \mathrm{a}$ \\
\hline Greece & $\mathrm{n} / \mathrm{a}$ & 7,824 & 10,108 & 14,695 & 5,734 & 10,719 & 11,680 & 7,480 & 6,844 \\
\hline Canada & 14,214 & 15,121 & 16,137 & 14,759 & 15,573 & 19,479 & 18,486 & 14,898 & 18,137 \\
\hline Argentina & $\mathrm{n} / \mathrm{a}$ & 85,340 & 50,287 & 37,743 & 37,531 & 30,344 & 61,671 & 53,555 & 45,381 \\
\hline
\end{tabular}


As is evidenced by the three tables above, the numbers of trademark registrations in developed countries exceeds, by far, trademark registrations in Arab countries and other developing countries. ${ }^{46}$ While in developed countries tens of thousands of trademarks are registered each year, in Arab countries and other developing countries, only a few thousand trademarks are registered annually. This gap between developed and developing countries has been expanding. Suffice it to consider the number of trademark applications for the year 1998-2000 as detailed in the next table.

Table 4

The Absolute Level

Trademark Applications-In Selected Countries (1998-2000) $)^{47}$

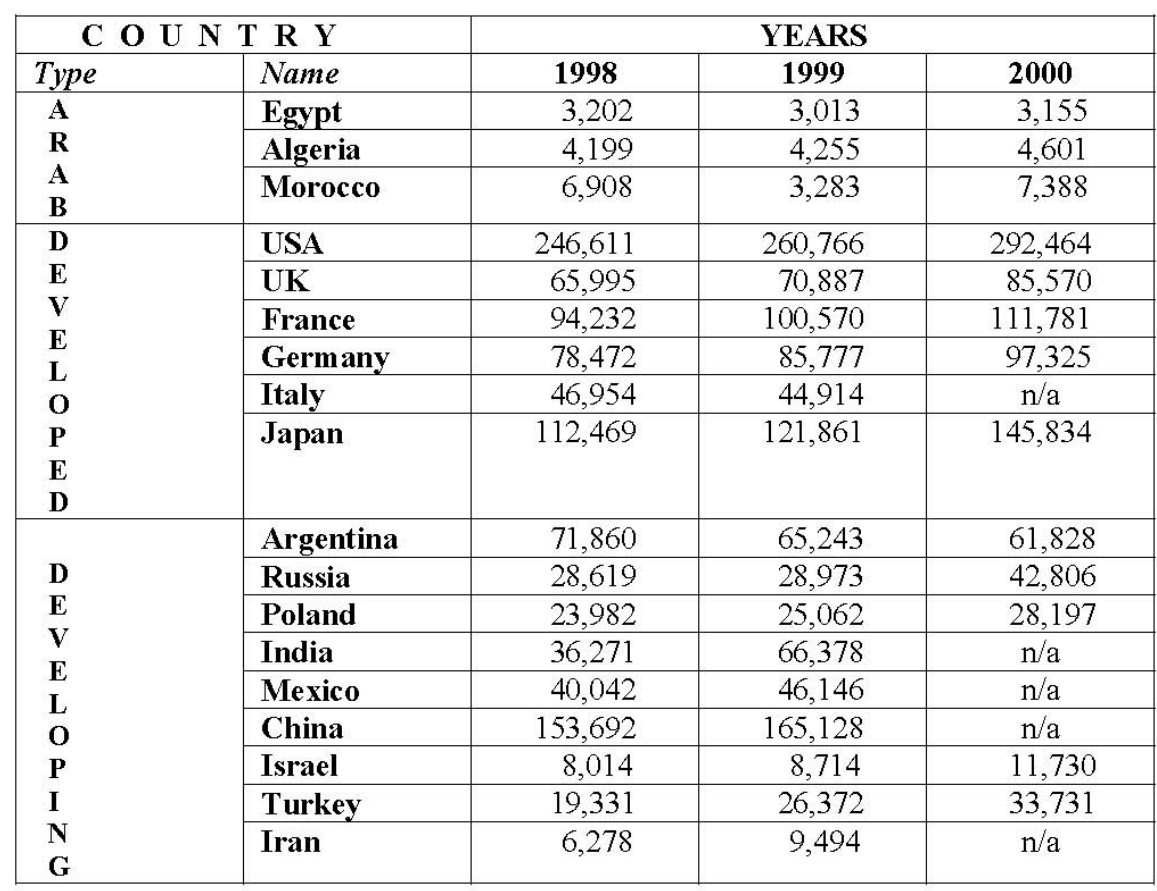

As alluded to above, the "Absolute" level provides an initial indication regarding the extent of trademark registration and use in a given country.

46. In tables 1, 2 and 3, "Developed Countries" include the U.S.; U.K.; France; Germany; Japan; Spain; and Canada. "Developing countries" include Arab countries; Israel; Turkey; India; South Korea; Brazil; Mexico; Greece and Argentina.

47. Based on statistics by WIPO. See www.wipo.int (last visited June 14, 2006). 
Marks that are not registered are not factored into these tables, because these marks (except for well-known marks) are not protected by trademark law and therefore, by definition, do not benefit from the existing trademark régime.

\subsection{The "Relative Level"}

The "Relative" level reveals the allocation of trademark registrations between local and foreign entities thus indicating the share of foreign trademark activity in a given country. Where foreign trademark owners own most of the trademarks that are registered in a given country, it is possible to argue that the trademark régime is used by foreigners. The next three tables detail "Relative" trademark registration in selected countries for the period 1973-2000. This is done by examining the ownership of trademark registrations by "Residents" and "Non-Residents."

Table 5

\section{The Relative Level}

\section{Trademark Registrations-In Selected Countries (1973-1982)}

\begin{tabular}{|c|c|c|c|c|c|c|c|c|c|c|c|}
\hline COUNTRY & TM-OWNER & 1973 & 1974 & 1975 & 1976 & 1977 & 1978 & 1979 & 1980 & 1981 & 1982 \\
\hline \multirow[t]{2}{*}{ Egypt } & Resident & 227 & 187 & 240 & 160 & 166 & 137 & 133 & 162 & $\mathrm{n} / \mathrm{a}$ & 124 \\
\hline & Non-Resident & 360 & 349 & 416 & 371 & 538 & 495 & 421 & 453 & $\mathrm{n} / \mathrm{a}$ & 415 \\
\hline \multirow[t]{2}{*}{ Syria } & Resident & 283 & 242 & 329 & $n / a$ & $\mathrm{n} / \mathrm{a}$ & 241 & 150 & 174 & 205 & 214 \\
\hline & Non-Resident & 513 & 432 & 491 & $\mathrm{n} / \mathrm{a}$ & $n / a$ & 708 & 475 & 1,026 & 504 & 548 \\
\hline \multirow[t]{2}{*}{ Jordan } & Resident & 67 & 22 & 40 & 40 & 36 & 113 & 70 & 134 & 90 & 37 \\
\hline & Non-Resident & 616 & 71 & 356 & 548 & 884 & 545 & 722 & 969 & 610 & 436 \\
\hline \multirow[t]{2}{*}{ Algeria } & Resident & 99 & 173 & 106 & 191 & 34 & 254 & 105 & 251 & 107 & 137 \\
\hline & Non-Resident & 684 & 967 & 397 & 491 & 195 & 1,171 & 475 & 430 & 665 & 2,070 \\
\hline \multirow[t]{2}{*}{ Israel } & Resident & 292 & 170 & 240 & 183 & 220 & 281 & 214 & 267 & 205 & 218 \\
\hline & Non-Resident & 1,186 & 1,006 & 1,088 & 900 & 938 & 883 & 863 & 918 & 756 & 862 \\
\hline \multirow[t]{2}{*}{ Turkey } & Resident & 753 & 593 & 557 & 617 & 830 & 1,262 & 1,129 & 1,475 & 1,287 & 1,377 \\
\hline & Non-Resident & 1,374 & 1,477 & 1,171 & 915 & 1,127 & 1,735 & 1,181 & 1,820 & 654 & 917 \\
\hline \multirow[t]{2}{*}{ U.S.A. } & Resident & 23,857 & 24,933 & 28,353 & 24,038 & 23,590 & 27,310 & 18,713 & 17,319 & 40,575 & 39,261 \\
\hline & Non-Resident & 2,255 & 2,448 & 2,578 & 2,288 & 2,268 & 2,320 & 1,772 & 1,566 & 2,127 & 3,183 \\
\hline \multirow[t]{2}{*}{ U.K. } & Resident & 6,458 & 5,984 & 5,878 & 6,287 & 5,106 & 5,489 & 5,133 & 3,356 & 4,673 & 6,169 \\
\hline & Non-Resident & 4,808 & 4,642 & 5,562 & 5,908 & 4,987 & 5,154 & 4,903 & 3,352 & 4,869 & 6,965 \\
\hline \multirow[t]{2}{*}{ France } & Resident & 19,657 & 19,631 & 12,645 & 10,951 & 36,461 & $n / a$ & $n / a$ & 37,332 & 26,201 & 28,628 \\
\hline & Non-Resident & 4,079 & 4,092 & 4,312 & 4,667 & 8,706 & $\mathrm{r} / \mathrm{a}$ & $n / a$ & 9,784 & 6,129 & 6,824 \\
\hline \multirow{2}{*}{$\begin{array}{l}\text { Germany } \\
\text { (Federal) }\end{array}$} & Resident & 9,768 & 9,712 & 9,396 & 9,445 & 10,018 & 10,434 & 10,776 & 13,006 & 11,241 & 11,601 \\
\hline & Non-Resident & 2,483 & 3,096 & 3,432 & 3,273 & 3,332 & 3,421 & 3,441 & 3,838 & 3,458 & 3,485 \\
\hline \multirow[t]{2}{*}{ Japan } & Resident & 68,099 & 86,646 & 104,156 & 90,821 & $n / a$ & $17 / \mathrm{a}$ & $n / a$ & $n / a$ & 63,940 & $n / a$ \\
\hline & Non-Resident & 3,789 & 3,856 & 5,010 & 5,124 & $\mathrm{n} / \mathrm{a}$ & $\mathrm{T} / \mathrm{a}$ & $n / a$ & $\mathrm{n} / \mathrm{a}$ & 4,374 & $\mathrm{n} / \mathrm{a}$ \\
\hline \multirow[t]{2}{*}{ Spain } & Resident & n/a & 12,387 & 13,112 & 21,235 & 29,187 & 35,644 & 41,434 & 16,241 & 14,693 & 17,065 \\
\hline & Non-Resident & $\mathrm{r} / \mathrm{a}$ & 1,588 & 1,550 & 1,971 & 3,458 & 2,710 & 3,912 & 1,911 & 2,073 & 2,310 \\
\hline \multirow[t]{2}{*}{ India } & Resident & 2,603 & 2,491 & 3,019 & 3,835 & 3,326 & 3,312 & 2,840 & n/a & 2,632 & 2,660 \\
\hline & Non-Resident & 955 & 812 & 640 & 921 & 878 & 713 & 512 & $n / a$ & 458 & 570 \\
\hline \multirow[t]{2}{*}{ S. Korea } & Resident & 2,622 & 1,578 & 1,460 & 2,958 & 2,618 & 5,369 & 4,486 & 4,665 & 3,966 & 4,420 \\
\hline & Non-Resident & 4,386 & 4,377 & 1,490 & 1,461 & 1,371 & 2,135 & 2,520 & 3,180 & 2,803 & 3,373 \\
\hline \multirow[t]{2}{*}{ Argentina } & Resident & 14,403 & 11,437 & 12,428 & 17,140 & 18,125 & 16,969 & 15,763 & 15,065 & 9,170 & 9,249 \\
\hline & Non-Resident & 3,622 & 2,664 & 2,032 & 2,109 & 2,214 & 2,618 & 3,268 & 4,235 & 3,201 & 2,982 \\
\hline
\end{tabular}

48. See WIPO, Intellectual Property Statistics, WIPO, Geneva, seewww.wipo.int (data on file with author) (last visited June 4, 2006). The term "Non-Residents" includes any legal entity that owns a trademark registration in the country but is not domiciled/based in that country. Thus, for example a Saudi company that owns a trademark registration in Jordan, would be considered a "Non-Resident" registrant in Jordan. 
Table 6

The Relative Level

\section{Trademark Registrations-In Selected Countries}

(1984-1991)

\begin{tabular}{|c|c|c|c|c|c|c|c|c|c|}
\hline COUNTRY & TM Owner & 1984 & 1985 & 1986 & 1987 & 1988 & 1989 & 1990 & 1991 \\
\hline \multirow{2}{*}{ Egypt } & Resident & 125 & $\mathrm{n} / \mathrm{a}$ & 175 & $\mathrm{n} / \mathrm{a}$ & 189 & $\mathrm{n} / \mathrm{a}$ & $\mathrm{n} / \mathrm{a}$ & 221 \\
\hline & Non-Resident & 525 & $\mathrm{n} / \mathrm{a}$ & 519 & $\mathrm{n} / \mathrm{a}$ & 554 & $\mathrm{n} / \mathrm{a}$ & $\mathrm{n} / \mathrm{a}$ & 673 \\
\hline \multirow[t]{2}{*}{ Jordan } & Resident & $\mathrm{n} / \mathrm{a}$ & $\mathrm{n} / \mathrm{a}$ & $\mathrm{n} / \mathrm{a}$ & $\mathrm{n} / \mathrm{a}$ & $n / a$ & 387 & $\mathrm{n} / \mathrm{a}$ & $\mathrm{n} / \mathrm{a}$ \\
\hline & Non-Resident & $\mathrm{n} / \mathrm{a}$ & $\mathrm{n} / \mathrm{a}$ & $n / a$ & $n / a$ & $\mathrm{n} / \mathrm{a}$ & 608 & $\mathrm{n} / \mathrm{a}$ & $\mathrm{n} / \mathrm{a}$ \\
\hline \multirow[t]{2}{*}{ S. Arabia } & Resident & $n / a$ & 292 & $\mathrm{n} / \mathrm{a}$ & $n / a$ & 311 & 298 & $\mathrm{IV} / \mathrm{a}$ & 384 \\
\hline & Non-Resident & $\mathrm{n} / \mathrm{a}$ & 1,464 & $\mathrm{n} / \mathrm{a}$ & $\mathrm{n} / \mathrm{a}$ & 1,514 & 1,765 & $\mathrm{n} / \mathrm{a}$ & 1,857 \\
\hline \multirow[t]{2}{*}{ Morocco } & Resident & 826 & 827 & 848 & 1,352 & 1,403 & 1,236 & 1,228 & 1,446 \\
\hline & Non-Resident & 470 & 437 & 483 & 554 & 583 & 569 & 681 & 621 \\
\hline \multirow[t]{2}{*}{ Sudan } & Resident & 81 & $\mathrm{n} / \mathrm{a}$ & $n / a$ & $n / a$ & $\mathrm{n} / \mathrm{a}$ & $\mathrm{n} / \mathrm{a}$ & $n / a$ & $\mathrm{n} / \mathrm{a}$ \\
\hline & Non-Resident & 424 & $\mathrm{n} / \mathrm{a}$ & $n / a$ & $n / a$ & $\mathrm{n} / \mathrm{a}$ & $\mathrm{n} / \mathrm{a}$ & $\mathrm{n} / \mathrm{a}$ & $\mathrm{n} / \mathrm{a}$ \\
\hline \multirow[t]{2}{*}{ Algeria } & Resident & 523 & 227 & 526 & 276 & 320 & 447 & 425 & 516 \\
\hline & Non-Resident & 1,769 & 873 & 1,372 & 3,497 & 3,584 & 3,833 & 3,869 & 3,690 \\
\hline \multirow[t]{2}{*}{ Tunisia } & Resident & 128 & 227 & 357 & $\mathrm{n} / \mathrm{a}$ & $n / \mathrm{a}$ & 259 & 315 & 425 \\
\hline & Non-Resident & 352 & 326 & 368 & $n / a$ & $\pi / a$ & 1,115 & 1,116 & 1,565 \\
\hline \multirow[t]{2}{*}{ Bahrain } & Resident & 27 & 46 & 67 & $n / \mathrm{a}$ & $n / \mathrm{a}$ & $n / \mathrm{a}$ & $\mathrm{n} / \mathrm{a}$ & $n / \mathrm{a}$ \\
\hline & Non-Resident & 833 & 582 & 540 & $\mathrm{n} / \mathrm{a}$ & $\mathrm{n} / \mathrm{a}$ & $\mathrm{n} / \mathrm{a}$ & $\mathrm{I} / \mathrm{a}$ & $\mathrm{r} / \mathrm{a}$ \\
\hline \multirow[t]{2}{*}{ Israel } & Resident & 155 & 131 & 310 & 200 & 270 & 280 & 400 & 504 \\
\hline & Non-Resident & 831 & 536 & 1,074 & 727 & 1,156 & 980 & 1,150 & 1,618 \\
\hline \multirow[t]{2}{*}{ Turkey } & Resident & 2,391 & $\mathrm{n} / \mathrm{a}$ & 3,137 & 3,271 & 3,390 & 3,300 & $\mathrm{n} / \mathrm{a}$ & 4,778 \\
\hline & Non-Resident & 747 & $\mathrm{n} / \mathrm{a}$ & 1,152 & 1,286 & 1,483 & 1,784 & $\mathrm{Il} / \mathrm{a}$ & 2,334 \\
\hline \multirow[t]{2}{*}{ U.S.A. } & Resident & 47,886 & 47,012 & $n / a$ & 44,754 & 44,755 & 52,246 & 50,642 & 38,486 \\
\hline & Non-Resident & 7,613 & 7,474 & $\mathrm{n} / \mathrm{a}$ & 8,779 & 8,474 & 10,237 & 10,701 & 8,161 \\
\hline \multirow[t]{2}{*}{ U.K. } & Resident & 7,773 & 7,901 & 8,463 & 7,176 & 7,710 & 12,349 & 14,700 & 15,395 \\
\hline & Non-Resident & 8,310 & 8,253 & 8,626 & 7,225 & 6,671 & 10,025 & 13,689 & 15,026 \\
\hline \multirow[t]{2}{*}{ France } & Resident & 31,370 & 30,539 & 38,302 & 46,437 & 49,093 & 50,031 & 52,811 & 64,425 \\
\hline & Non-Resident & 7,218 & 6,653 & 19,847 & 17,764 & 21,488 & 24,688 & 26,241 & 26,124 \\
\hline \multirow[t]{2}{*}{ Germany } & Resident & 10,696 & 10,112 & 10,673 & 11,112 & 11,697 & 13,680 & $\mathrm{n} / \mathrm{a}$ & $\mathrm{n} / \mathrm{a}$ \\
\hline & Non-Resident & 3,562 & 3,403 & 7,058 & 7,575 & 8,491 & 10,217 & $n / \mathrm{a}$ & $\Pi / \mathrm{a}$ \\
\hline \multirow[t]{2}{*}{ Japan } & Resident & 107,153 & 110,146 & 109,456 & 108,300 & 109,994 & 109,577 & 107,318 & 87,231 \\
\hline & Non-Resident & 9,483 & 9,388 & 9,019 & 8,775 & 9,293 & 10,021 & 9,867 & 8,098 \\
\hline \multirow[t]{2}{*}{ Spain } & Resident & 9,198 & 12,891 & 14,724 & 20,072 & 19,455 & 21,252 & 31,388 & 39,932 \\
\hline & Non-Resident & 1,865 & 1,806 & 2,210 & 2,821 & 2,659 & 2,411 & 4,632 & 5,159 \\
\hline \multirow[t]{2}{*}{ India } & Resident & 1,797 & 1,579 & 2,522 & 2,513 & 3,404 & 4,431 & 5,184 & 6,025 \\
\hline & Non-Resident & 443 & 291 & 443 & 562 & 962 & 904 & 1,245 & 1,400 \\
\hline \multirow[t]{2}{*}{ Brazil } & Resident & 13,754 & 18,435 & 14,921 & 19,102 & 30,746 & $\mathrm{n} / \mathrm{a}$ & 30,131 & 19,007 \\
\hline & Non-Resident & 3,945 & 3,136 & 2,136 & 2,238 & 2,452 & $n / \mathrm{a}$ & 4,183 & 3,326 \\
\hline \multirow[t]{2}{*}{ Mexico } & Resident & 3,656 & 3,290 & 3,495 & 9,835 & 9,557 & 7,962 & 10,134 & 8,026 \\
\hline & Non-Resident & 4,166 & 3,678 & 4,119 & 8,989 & 7,774 & 5,830 & 7,404 & 6,211 \\
\hline \multirow[t]{2}{*}{ Greece } & Resident & 1,371 & 1,846 & $\mathrm{n} / \mathrm{a}$ & 2,654 & $\mathrm{n} / \mathrm{a}$ & $n / \mathrm{a}$ & $\mathrm{n} / \mathrm{a}$ & $\mathrm{n} / \mathrm{a}$ \\
\hline & Non-Resident & 1,463 & 1,971 & $\mathrm{n} / \mathrm{a}$ & 2,978 & $\mathrm{n} / \mathrm{a}$ & $n / \mathrm{a}$ & $\mathrm{n} / \mathrm{a}$ & $\mathrm{n} / \mathrm{a}$ \\
\hline \multirow[t]{2}{*}{ Canada } & Resident & 7,096 & 6,742 & 6,987 & 7,615 & 7,875 & 8,539 & 7,659 & 8,049 \\
\hline & Non-Resident & 5,109 & 4,494 & 5,336 & 6,103 & 5,803 & 5,823 & 6,099 & 6,548 \\
\hline \multirow[t]{2}{*}{ Argentina } & Resident & $\mathrm{n} / \mathrm{a}$ & $\mathrm{n} / \mathrm{a}$ & $\mathrm{n} / \mathrm{a}$ & $\mathrm{n} / \mathrm{a}$ & 54,549 & $\mathrm{n} / \mathrm{a}$ & $\mathrm{n} / \mathrm{a}$ & $\mathrm{n} / \mathrm{a}$ \\
\hline & Non-Resident & $\mathrm{n} / \mathrm{a}$ & $\mathrm{n} / \mathrm{a}$ & $\mathrm{n} / \mathrm{a}$ & $\mathrm{n} / \mathrm{a}$ & 8,300 & $\mathrm{n} / \mathrm{a}$ & $\mathrm{n} / \mathrm{a}$ & $\mathrm{n} / \mathrm{a}$ \\
\hline
\end{tabular}

*Federal Republic of Germany 
Table 7

The Relative Level

Trademark Registrations-In Selected Countries

(1992-2000)

\begin{tabular}{|c|c|c|c|c|c|c|c|c|c|c|}
\hline COUNTRY & TM Owner & 1992 & 1993 & 1994 & 1995 & 1996 & 1997 & 1998 & 1999 & 2000 \\
\hline \multirow{2}{*}{ Egypt } & Resident & $\mathrm{n} / \mathrm{a}$ & $n / a$ & $\mathrm{n} / \mathrm{a}$ & $\mathrm{n} / \mathrm{a}$ & $\mathrm{n} / \mathrm{a}$ & $\mathrm{n} / \mathrm{a}$ & $\mathrm{n} / \mathrm{a}$ & $\mathrm{n} / \mathbf{a}$ & $\mathrm{n} / \mathrm{a}$ \\
\hline & Non-Resident & $n / \mathbf{a}$ & n/a & $n / a$ & $n / a$ & 2,285 & 2,630 & 2,841 & 2,690 & 3,155 \\
\hline \multirow[t]{2}{*}{ Jordan } & Resident & $n / a$ & $\pi / \mathbf{a}$ & 1,215 & 2,462 & 2,200 & 2,159 & 899 & 891 & 876 \\
\hline & Non-Resident & $\mathrm{n} / \mathrm{a}$ & $\mathrm{n} / \mathrm{a}$ & 923 & 1,070 & 1,800 & 1,926 & 1,453 & 1,603 & 1,660 \\
\hline \multirow{2}{*}{ Morocco } & Resident & 1,549 & 2,776 & 3,403 & 2,711 & 4,500 & $\mathrm{n} / \mathrm{a}$ & 2,368 & $n / a$ & $\mathrm{n} / \mathrm{a}$ \\
\hline & Non-Resident & 799 & 1,444 & 1,299 & 1,853 & 5,154 & 3,312 & 4,540 & 3,283 & 3,579 \\
\hline \multirow[t]{2}{*}{ Algeria } & Resident & 455 & 738 & 507 & 592 & 661 & $\mathrm{n} / \mathrm{a}$ & 526 & 769 & 1,248 \\
\hline & Non-Resident & 4,660 & 5,061 & 4,733 & 4,256 & 3,581 & 2,362 & 3,176 & 3,143 & 3,317 \\
\hline \multirow[t]{2}{*}{ Sudan } & Resident & $n / \mathbf{a}$ & $\pi / a$ & $\pi / a$ & $n / a$ & 0 & 0 & 0 & 0 & 0 \\
\hline & Non-Resident & $\mathrm{n} / \mathrm{a}$ & $n / a$ & $\mathrm{n} / \mathrm{a}$ & $n / a$ & 1,508 & 1,482 & 1,514 & 1,283 & 1,243 \\
\hline \multirow[t]{2}{*}{ Tunisia } & Resident & $n / a$ & 1.988 & 654 & 903 & 984 & $\mathrm{n} / \mathrm{a}$ & $\mathrm{n} / \mathrm{a}$ & $\mathrm{n} / \mathrm{a}$ & $\mathrm{n} / \mathrm{a}$ \\
\hline & Non-Resident & $n / \mathbf{a}$ & 2,605 & 1,700 & 1,816 & 1,900 & $\mathrm{n} / \mathrm{a}$ & $\mathrm{n} / \mathrm{a}$ & $n / a$ & $n / a$ \\
\hline \multirow{2}{*}{ Bahrain } & Resident & 102 & $\mathrm{n} / \mathrm{a}$ & $\mathrm{n} / \mathrm{a}$ & $\mathrm{n} / \mathrm{a}$ & $n / a$ & $\mathrm{n} / \mathrm{a}$ & $\mathrm{n} / \mathrm{a}$ & $\mathrm{n} / \mathrm{a}$ & $n / a$ \\
\hline & Non-Resident & 636 & $n / \mathbf{a}$ & $n / a$ & $n / a$ & $n / a$ & $n / a$ & $n / a$ & $n / a$ & $n / a$ \\
\hline \multirow[t]{2}{*}{ Israel } & Resident & 441 & 747 & 1,016 & 1,505 & 1,393 & 1,467 & 1,646 & 1,572 & 1,753 \\
\hline & Non-Resident & 1,713 & 2,624 & 3,561 & 4,131 & 4,389 & 5,556 & 5,571 & 5,423 & 5,672 \\
\hline \multirow{2}{*}{ Turkey } & Resident & 4,444 & 5,549 & 6,760 & 3,855 & 4,684 & 7,850 & 5,427 & 12,019 & 10,438 \\
\hline & Non-Resident & 2,741 & 2,288 & 2,586 & 1,590 & 2,518 & 3,471 & 2,037 & 8,382 & 10,437 \\
\hline \multirow[t]{2}{*}{ U.S.A. } & Resident & 68,201 & 67,238 & 54,186 & 72,796 & 80,874 & 108,071 & 98,526 & 76,082 & 95,448 \\
\hline & Non-Resident & 11,972 & 13,376 & 9,717 & 12,761 & 12,840 & 30,084 & 31,345 & 11,349 & 14,096 \\
\hline \multirow{2}{*}{ U.K. } & Resident & 16,092 & 15,011 & 13,376 & 15,729 & 29,169 & 27,897 & 29,563 & 33,309 & 44,292 \\
\hline & Non-Resident & 19,045 & 17,923 & 15,452 & 17,671 & 28,274 & 21,138 & 19,037 & 18,132 & 21,357 \\
\hline \multirow[t]{2}{*}{ France } & Resident & 64,857 & 68,666 & 53,900 & 38,100 & 46,400 & 47,798 & 71,491 & 80,020 & 89,850 \\
\hline & Non-Resident & 24,166 & 23,566 & 24,874 & 22,612 & 16,069 & 15,077 & 22,741 & 20,550 & 21,931 \\
\hline \multirow[t]{2}{*}{ Germany" } & Resident & $\mathrm{n} / \mathrm{a}$ & $\mathrm{n} / \mathrm{a}$ & 26,489 & 16,285 & 24,464 & 40,631 & 44,497 & 48,021 & 55,680 \\
\hline & Non-Resident & $n / \mathbf{a}$ & $n / \mathbf{a}$ & 18,718 & 5,649 & 15,792 & 16,804 & 13,421 & 13,018 & 14,599 \\
\hline \multirow[t]{2}{*}{ Japan } & Resident & 141,472 & 145,229 & 135,902 & 136,883 & 165,544 & 221,830 & 114,895 & 106,036 & 79,462 \\
\hline & Non-Resident & 14,568 & 13,456 & 11,289 & 8,028 & 12,707 & 31,442 & 17,171 & 17,620 & 17,648 \\
\hline \multirow[t]{2}{*}{ Spain } & Resident & 74,884 & 52,163 & 54,883 & 60,062 & 63,434 & 46,976 & 49,295 & 53,014 & 58,860 \\
\hline & Non-Resident & 9,332 & 7,163 & 8,786 & 9,580 & 17,583 & 12,398 & 10,515 & 10,072 & 15,059 \\
\hline \multirow{2}{*}{ India } & Resident & 5,033 & 4,706 & 4,688 & 5,310 & 3,654 & $\mathrm{n} / \mathrm{a}$ & 4,302 & 6,747 & $\mathrm{n} / \mathrm{a}$ \\
\hline & Non-Resident & 957 & 496 & 958 & $n / a$ & 782 & $\mathrm{n} / \mathrm{a}$ & 538 & 1,263 & $n / \mathbf{a}$ \\
\hline \multirow[t]{2}{*}{ Brazil } & Resident & 32,184 & 23,712 & 18,270 & 21,076 & 24,985 & $\pi / \mathrm{a}$ & $n / a$ & $n / a$ & $\pi / \mathbf{a}$ \\
\hline & Non-Resident & 5,780 & 4,969 & 4,524 & 4,254 & 6,217 & $\mathrm{n} / \mathrm{a}$ & $\mathrm{n} / \mathrm{a}$ & $\mathrm{n} / \mathrm{a}$ & $n / a$ \\
\hline \multirow[t]{2}{*}{ Mexico } & Resident & 13,632 & 11,341 & 17,849 & 15,229 & 14,451 & 16,761 & 16,775 & 23,423 & $n / a$ \\
\hline & Non-Resident & 11,188 & 9,100 & 15,868 & 14,729 & 11,305 & 11,060 & 11,587 & 16,891 & $n / a$ \\
\hline \multirow{2}{*}{ Greece } & Resident & $\mathrm{n} / \mathrm{a}$ & 3,015 & 3,297 & 5,344 & 2,122 & 4,043 & 4,615 & 3,977 & 3,191 \\
\hline & Non-Resident & $n / a$ & 4,809 & 6,816 & 9,351 & 3,612 & 6,676 & 7,065 & 3,503 & 3,653 \\
\hline \multirow[t]{2}{*}{ Canada } & Resident & 7,370 & 7,802 & 8,179 & 7,688 & 7,928 & 10,239 & 10,331 & 7,966 & 9,462 \\
\hline & Non-Resident & 6,844 & 7,319 & 7,198 & 7,171 & 7,645 & 9,240 & 8,155 & 6,932 & 8,675 \\
\hline \multirow[t]{2}{*}{ Argentina } & Resident & $n / a$ & 66,202 & $\mathrm{n} / \mathrm{a}$ & $n / a$ & n/a & $\mathrm{n} / \mathrm{a}$ & 42,661 & 37,844 & 31,430 \\
\hline & Non-Resident & $n / a$ & 19,138 & $\mathrm{n} / \mathrm{a}$ & $n / a$ & $n / a$ & $\mathrm{n} / \mathrm{a}$ & 19,010 & 15,711 & 13,951 \\
\hline
\end{tabular}

*Federal Republic of Germany

The information depicted in tables 5-7 reveals that at the "Relative" level there is a visible difference between developed and developing countries. While in developed countries residents own the majority of registered trademarks, non-residents dominate trademark registration in Arab countries. Thus, it appears that while in developed countries local business and brand owners are utilizing the trademark system by registering their brands, trademark registration by residents of developing countries is rather dormant and in some cases negligible. For example, in 1989 a total of 2,063 marks 
were registered in the Kingdom of Saudi Arabia, but only 298 of those marks were registered by residents of that country. Similarly, in the year 2000, 2,536 marks were registered in Jordan but only on third of those (876) were registered by residents of Jordan. In contrast, during 1998 Japan's trademark office registered 126,656 marks and only 17,620 of those were owned by nonresidents of Japan. The rest of those registered marks (a staggering 106,036) were owned by residents of Japan.

It should be noted that similar trends have been observed during the late 1970s. According to a 1977 report by UNCTAD, the share of foreign-owned trademarks registered in different world regions for the years 1964 and 1974 was much higher in developing regions than in developed regions. Specifically, Africa was the continent with the highest share of foreign owned trademarks registered therein ( $88 \%$ of all trademarks registered in Africa). Asia, followed suit with $65 \%$ and Latin America had 34\%. Significantly, the UNCTAD report indicates that in developing countries, as a whole, the share of non-residents in trademark registrations had risen significantly from $27 \%$ in 1964 to about $50 \%$ in 1974 . In contrast, developed market economies had the lowest share of foreign owned trademark registrations. ${ }^{49}$

From all of this, it is possible to conclude that the pattern of trademark registration in both developed and developing countries has remained constant despite the introduction of modern trademark laws that are TRIPS compliant.

\subsection{The "Particular Level"}

After concluding that a substantial portion of trademarks registered in Arab countries are registered by foreign (non-resident) entities. The compelling question would be: from which countries do these foreign marks originate? Indeed, the "Particular" level goes a step ahead of the "Relative" level and examines the specific national identity of the "non-resident" brand owners. In essence, this level transcends the "Relative" level by revealing the countries whose residents dominate trademark registration in other countries.

49. UNCTAD, The Role of the Patent System in the Transfer of Technology to Developing Countries, United Nations Publication, No. E. 75 II. D. 6, at 15 (June 1977). According to that report, the term "Developing Countries" refers to regions: Africa, Asia and Latin America. Arab countries may be included in either Africa or Asia. Also see WIPO, Industrial Property Statistics for 1974 (IP/STAT/ 1974/3), Geneva, Dec. 1976. UNCTAD, id. at 15-16. The UNCTAD report points to western countries in which there was a high share of foreigners that have registered trademarks therein: e.g. Norway (84\%); Finland (81\%); Australia (59\%); Greece (53\%); and Benelux countries (44\%). But these foreign-owned marks originate in developed countries. Interestingly, the report indicated that in 1972, foreigners owned $84 \%$ of all patents granted in developing countries. 
This issue is of great significance because, if residents of Arab countries own "foreign" trademarks, registered in an Arab country, then it cannot be argued that Arab countries are not making use of the trademark system. On the other hand, if it is determined that foreign marks registered in Arab countries are owned by residents of developed countries, then, it would be possible to conclude that the trademark régime in Arab countries is primarily intended to protect brands originating in developed countries.

As early as 1979, a report by UNCTAD indicated that trademarks in developing countries are largely owned by nationals of developed (industrialized) countries. According to that report, trademarks were "increasingly being registered by foreigners, most of them located in developed countries." 50 The report concluded that $97 \%$ of all trademarks registered by foreigners had originated in developed market-economy countries, whereas nationals of developing countries owned only $2.2 \%$ of all foreign trademarks worldwide. Furthermore, the report concluded that $96.9 \%$ of all marks registered by foreign entities in developed countries as well as developing countries originated in developed countries.

Specifically, in $1974,34 \%$ of all foreign registered trademarks in developing countries were owned by U.S. corporations. Furthermore, according to that report, the other four leading industrial countries (Japan, U.K., France and Federal Republic of Germany) collectively accounted for $43 \%$ of trademark registrations in developing countries. Indeed, only about 2,000 trademarks, originating in developing countries, were registered outside of their home countries in 1974. In addition, in that year, the flow of marks from developing countries to developed countries constituted only 1.1 of all foreign marks registered in developed countries. ${ }^{51}$ The same report also provides some indication as to the nature of foreign trademarking activity of different countries. The report states that in 1974, $43.8 \%$ of all marks originating in developed countries were registered in developing countries. In contrast, $72 \%$ of marks originating in developing countries were registered in developing countries. Thus, only $28 \%$ of marks originating in developing countries are registered in developed countries. In other words, marks originating in developing countries are primarily used in other developing countries.

Clearly, not only do developed countries "host" relatively fewer foreign trademark registrations in their respective jurisdictions, their trademarks are

50. Id. at 15 .

51. Id. at $15-16$ 
spread out among all regions of the world. On the other hand, developing countries typically have a larger portion of trademark registrations by nonresidents and their residents are inclined to limit their external trademarking activity to other developing countries and not to venture into developed countries. Thus, while brand owners in developed countries are heavily involved in trademarking activity within developing countries, the opposite is not true. This indicates that brand owners from developing countries do not (proportionally) venture into developing markets in order to sell their branded goods/services. Significantly, the findings in that research which was conducted in the late 1970s have remained constant through the end of 1999. The next table depicts the national origin of "non-resident" trademarks in selected countries around the world for the year 1999. ${ }^{52}$

Table 8

National Origin of Some Foreign (Non-Resident) Trademark Applications in Selected Countries Around the World (1999)

\begin{tabular}{|c|c|c|c|c|c|c|c|c|c|c|c|c|c|c|}
\hline \multicolumn{2}{|c|}{ Country } & \multicolumn{12}{|c|}{ COUNTRY OF ORIGIN OF TRADEMARK APPLICANTS $^{53}$} & \multirow[b]{2}{*}{$\begin{array}{l}\text { total } \\
\text { no. of } \\
\text { apps. } \\
\text { filed } \\
\text { by } \\
\text { non- } \\
\text { res. }\end{array}$} \\
\hline Type & Name & TR & $\mathbf{U K}$ & FR & IT & DE & JP & RU & $\mathrm{CN}$ & $\mathbf{I N}$ & MX & IL & USA & \\
\hline \multirow{4}{*}{$\begin{array}{l}\mathbf{A} \\
\mathbf{R} \\
\mathbf{A} \\
\mathbf{B}\end{array}$} & Algeria & 30 & 113 & 711 & 260 & 702 & 67 & 32 & 52 & $n / \mathrm{a}$ & $\Pi / \mathrm{a}$ & $n / a$ & 335 & 3310 \\
\hline & Egypt & $\mathrm{n} / \mathrm{a}$ & $\mathrm{n} / \mathrm{a}$ & 681 & 378 & 850 & $\mathrm{n} / \mathrm{a}$ & 34 & 55 & $\mathrm{n} / \mathrm{a}$ & $\mathrm{n} / \mathrm{a}$ & $n / a$ & $\mathrm{n} / \mathrm{a}$ & 690 \\
\hline & Morocco & $\mathrm{n} / \mathrm{a}$ & 1 & 1039 & 327 & 750 & $\mathrm{n} / \mathrm{a}$ & 32 & 36 & $\mathrm{n} / \mathrm{a}$ & $\mathrm{n} / \mathrm{a}$ & $\mathrm{n} / \mathrm{a}$ & $\mathrm{n} / \mathrm{a}$ & 3283 \\
\hline & Sudan & $\mathrm{n} / \mathrm{a}$ & $\mathrm{n} / \mathrm{a}$ & 239 & 159 & 422 & $\mathrm{n} / \mathrm{a}$ & 28 & 35 & $\mathrm{n} / \mathrm{a}$ & $\mathrm{n} / \mathrm{a}$ & $\mathrm{n} / \mathrm{a}$ & $\mathrm{n} / \mathrm{a}$ & 1283 \\
\hline \multirow{9}{*}{$\begin{array}{l}\text { D } \\
\mathbf{E} \\
\text { V } \\
\mathbf{E} \\
\mathbf{L} \\
\mathrm{O} \\
\mathbf{P} \\
\mathbf{E} \\
\mathrm{D}\end{array}$} & U.S.A. & 35 & 3044 & 2272 & 1270 & 3548 & 2374 & 65 & 291 & 134 & 662 & 619 & ----- & 31045 \\
\hline & U.K. & 33 & ---. & 2515 & 426 & 3839 & 798 & 25 & 89 & 63 & 32 & 43 & 5641 & 21062 \\
\hline & France & 46 & 1587 & --- & 795 & 4470 & 945 & 63 & 156 & 15 & 15 & 45 & 4492 & 20550 \\
\hline & Germany & 57 & 373 & 2666 & 788 & --- & 390 & 70 & 103 & 15 & 9 & 23 & 1888 & 14214 \\
\hline & Italy & $\mathrm{n} / \mathrm{a}$ & $\mathrm{n} / \mathrm{a}$ & 2383 & ---- & 3688 & $\mathrm{n} / \mathrm{a}$ & 39 & 79 & $\mathrm{n} / \mathrm{a}$ & $\mathrm{n} / \mathrm{a}$ & $\mathrm{n} / \mathrm{a}$ & $\mathrm{n} / \mathrm{a}$ & 17280 \\
\hline & Japan & 4 & 1069 & 1255 & 615 & 1892 & ---- & 14 & 124 & 11 & 24 & 83 & 8081 & 17609 \\
\hline & Canada & 5 & 883 & 1012 & 458 & 1258 & 650 & 10 & 96 & 23 & 79 & 76 & 12261 & 19863 \\
\hline & Spain & 32 & 478 & 2816 & 784 & 3684 & 288 & 41 & 118 & 16 & 38 & 16 & 1865 & 14918 \\
\hline & Finland & 18 & 157 & 1018 & 72 & 2217 & 71 & 18 & 46 & 1 & 7 & 5 & 645 & 6851 \\
\hline \multirow{7}{*}{$\begin{array}{l}\text { D } \\
\text { E } \\
\text { V } \\
\text { E } \\
\text { L } \\
\text { O } \\
\text { P } \\
\text { I } \\
\text { N } \\
\text { G }\end{array}$} & Turkey & --- & 344 & 1343 & 230 & 2610 & 129 & 35 & 51 & 5 & $\mathrm{n} / \mathrm{a}$ & 12 & 1269 & 8568 \\
\hline & Israel & 11 & 379 & 458 & 204 & 771 & 124 & 20 & 21 & 6 & 3 & ---- & 2797 & 6147 \\
\hline & China & 45 & 1260 & 1663 & 961 & 2729 & 2698 & 68 & --- & 45 & 7 & 55 & 8086 & 24508 \\
\hline & Colombia & $n / a$ & 382 & 421 & 156 & 587 & 157 & $n / a$ & 20 & 7 & 179 & 18 & 2661 & 6885 \\
\hline & Argentina & 4 & 1117 & 1429 & 760 & 1698 & 540 & 3 & 30 & 9 & 349 & 17 & 6927 & 18732 \\
\hline & India & 3 & 356 & 385 & 218 & 110 & 240 & 3 & 67 & ---- & $\mathrm{n} / \mathrm{a}$ & 12 & 2052 & 5393 \\
\hline & Mexico & 1 & 771 & 869 & 365 & 1447 & 384 & 4 & 46 & 13 & ---- & 28 & 8871 & 16657 \\
\hline
\end{tabular}

52. The information depicted in this table is based on: The World Intellectual Property Organization (WIPO), Applications for Registration Filed by Non-Residents in 1999, WIPO, IP/STAT/1999/B marks, www.wipo.int (last visited Aug. 29, 2006).

53. Initials of "Countries of Origin": TR=Turkey, UK=United Kingdom, FR=France, IT=Italy, $\mathrm{DE}=$ Germany, $\mathrm{JP}=\mathrm{Japan}, \mathrm{RU}=$ Russia, $\mathrm{CN}=\mathrm{China}, \mathrm{IN}=\mathrm{India}, \mathrm{MX}=$ Mexico, $\mathrm{IL}=\mathrm{Israel}, \mathrm{USA}=$ United States of America. 
The table above indicates that trademarks originating in developed countries (i.e. the U.S., U.K., France, Germany, Italy and Japan) dominate trademark registrations by "non-residents" in all the three country groups. Specifically, during 1999, a total of 2,690 trademark applications in Egypt were applied for by non-residents. Marks originating in France, Italy and Germany accounted for 1,909 of these. In other words residents of these countries owned $71 \%$ of foreign marks filed in Egypt during that year. Similarly, in the same year 64\% of foreign marks registered in Algeria, originated in the U.K., France, Italy, Germany and the U.S. Likewise, 64\% of foreign marks filed during 1999 in Morocco are owned by residents of France, Italy and Germany. This trend is, also, clearly manifested in the case of Jordan. The following table details the number and origin of trademarks registered in Jordan during eight successive years (1994-2001). 
Table 9

Trademark Registrations in the Hashemite Kingdom of Jordan (By Country of Origin) (1994-2001) ${ }^{54}$

\begin{tabular}{|c|c|c|c|c|c|c|c|c|c|c|}
\hline \multirow{2}{*}{$\begin{array}{l}\text { Country } \\
\text { Type }\end{array}$} & \multirow{2}{*}{ Full Name of Country } & \multicolumn{8}{|c|}{ YEARS (1994-1996) } & \multirow[t]{2}{*}{ Total } \\
\hline & & 1994 & 1995 & 1996 & 1997 & 1998 & 1999 & 2000 & 2001 & \\
\hline \multirow[b]{4}{*}{$\mathbf{A}$} & Jordan & 1215 & 1070 & 720 & 1010 & 899 & 891 & 876 & 788 & 7469 \\
\hline & Egypt & 13 & 5 & 6 & 0 & 3 & 9 & 3 & 4 & 43 \\
\hline & Saudi Arabia & 19 & 66 & 52 & 10 & 34 & 13 & 71 & 35 & 300 \\
\hline & Syria & 10 & 19 & 1 & 3 & 13 & 17 & 28 & 31 & 122 \\
\hline \multirow{3}{*}{$\mathbf{R}$} & United Arab Emirates & 0 & 10 & 2 & 9 & 14 & 21 & 23 & 25 & 104 \\
\hline & Lebanon & 7 & 6 & 1 & 11 & 4 & 13 & 19 & 17 & 78 \\
\hline & Morocco & 2 & 0 & 0 & 0 & 0 & 2 & 1 & 0 & 5 \\
\hline \multirow{2}{*}{$\mathbf{A}$} & Bahrain & 1 & 0 & 1 & 0 & 0 & 1 & 2 & 0 & 5 \\
\hline & Kuwait & 1 & 0 & 1 & 5 & 46 & 16 & 16 & 14 & 99 \\
\hline \multirow{5}{*}{ B } & Oman & 6 & 9 & 0 & 0 & 0 & 0 & 0 & 4 & 19 \\
\hline & Palestinian N. Authority & 0 & 1 & 4 & 6 & 4 & 0 & 4 & 5 & 24 \\
\hline & Qatar & 2 & 0 & 0 & 0 & 1 & 2 & 0 & 1 & 6 \\
\hline & Tunisia & 0 & 3 & 1 & 0 & 0 & 0 & 0 & 4 & 8 \\
\hline & Yemen & 0 & 0 & 0 & 0 & 1 & 0 & 1 & 0 & 2 \\
\hline \multirow{2}{*}{ D } & United States & 310 & 415 & 474 & 336 & 486 & 519 & 517 & 370 & 3427 \\
\hline & United Kingdom & 107 & 121 & 107 & 138 & 133 & 162 & 119 & 141 & 1028 \\
\hline \multirow{2}{*}{$\mathbf{E}$} & France & 74 & 145 & 142 & 75 & 144 & 147 & 151 & 103 & 981 \\
\hline & Germany & 55 & 116 & 168 & 88 & 104 & 161 & 205 & 146 & 1043 \\
\hline \multirow{3}{*}{$\mathbf{V}$} & Italy & 39 & 68 & 56 & 20 & 51 & 69 & 54 & 46 & 403 \\
\hline & Spain & 17 & 30 & 11 & 16 & 23 & 25 & 25 & 43 & 190 \\
\hline & Switzerland & 64 & 93 & 60 & 107 & 121 & 89 & 114 & 136 & 784 \\
\hline \multirow[t]{2}{*}{$\mathbf{E}$} & Canada & 4 & 0 & 3 & 1 & 9 & 11 & 5 & 9 & 42 \\
\hline & Japan & 49 & 67 & 55 & 26 & 33 & 68 & 74 & 57 & 429 \\
\hline \multirow{2}{*}{$\mathbf{L}$} & Hong Kong & 10 & 3 & 11 & 8 & 1 & 1 & 1 & 3 & 38 \\
\hline & Netherlands & 24 & 78 & 36 & 29 & 58 & 77 & 55 & 34 & 391 \\
\hline & Luxembourg & 2 & 2 & 5 & 5 & 6 & 17 & 7 & 2 & 46 \\
\hline O & Sweden & 8 & 13 & 11 & 7 & 14 & 3 & 14 & 7 & 77 \\
\hline & Liechtenstein & 2 & 3 & 2 & 5 & 9 & 57 & 2 & 11 & 91 \\
\hline $\mathbf{P}$ & Belgium & 3 & 3 & 4 & 6 & 5 & 17 & 14 & 6 & 58 \\
\hline & Finland & 9 & 1 & 4 & 3 & 3 & 1 & 5 & 9 & 35 \\
\hline $\mathbf{E}$ & Singapore & 1 & 3 & 3 & 7 & 5 & 2 & 2 & 2 & 25 \\
\hline & Ireland & 1 & 3 & 12 & 6 & 0 & 0 & 2 & 8 & 32 \\
\hline & Channel Islands & 14 & 10 & 14 & 2 & 0 & 5 & 1 & 1 & 47 \\
\hline $\mathbf{D}$ & Scotland & 2 & 1 & 1 & 3 & 0 & 0 & 0 & 1 & 8 \\
\hline & South Korea & 14 & 34 & 45 & 13 & 31 & 12 & 12 & 11 & 72 \\
\hline & South Africa & 0 & 0 & 8 & 11 & 7 & 0 & 0 & 1 & 27 \\
\hline & Taiwan & 6 & 8 & 8 & 0 & 0 & 2 & 1 & 2 & 27 \\
\hline D & Brazil & 5 & 13 & 8 & 2 & 2 & 4 & 2 & 2 & 38 \\
\hline & Argentina & 0 & 7 & 6 & 1 & 0 & 1 & 3 & 1 & 19 \\
\hline & China & 7 & 6 & 7 & 4 & 6 & 8 & 12 & 10 & 60 \\
\hline $\mathbf{E}$ & Israel & 0 & 0 & 9 & 24 & 9 & 5 & 5 & 3 & 55 \\
\hline & Indonesia & 6 & 0 & 1 & 0 & 8 & 1 & 0 & 11 & 27 \\
\hline V & India & 4 & 13 & 2 & 4 & 4 & 6 & 4 & 5 & 42 \\
\hline & North Korea & 3 & 1 & 0 & 0 & 0 & 1 & 0 & 0 & 5 \\
\hline $\mathbf{E}$ & Colombia & 0 & 0 & 0 & 0 & 5 & 4 & 1 & 0 & 10 \\
\hline & Mexico & 7 & 1 & 9 & 5 & 0 & 2 & 0 & 1 & 25 \\
\hline & Saline & 0 & 0 & 1 & 0 & 4 & 1 & 2 & 0 & 8 \\
\hline $\mathbf{L}$ & Turkey & 3 & 4 & 6 & 4 & 38 & 12 & 48 & 9 & 124 \\
\hline & Thailand & 0 & 1 & 0 & 2 & 4 & 1 & 2 & 6 & 16 \\
\hline O & Russia & 0 & 0 & 2 & 0 & 0 & 5 & 0 & 0 & 7 \\
\hline & Malaysia & 0 & 4 & 0 & 6 & 0 & 2 & 0 & 1 & 13 \\
\hline $\mathbf{P}$ & Pakistan & 0 & 0 & 0 & 0 & 1 & 0 & 0 & 0 & 1 \\
\hline & Slovenia & 1 & 1 & 3 & 0 & 0 & 0 & 0 & 0 & 5 \\
\hline $\mathbf{I}$ & Uruguay & 0 & 0 & 0 & 0 & 0 & 0 & 1 & 0 & 1 \\
\hline $\boldsymbol{I}$ & Sri Lanka & 2 & 0 & 0 & 0 & 0 & 0 & 2 & 5 & 9 \\
\hline & Bahamas & 0 & 0 & 0 & 2 & 3 & 1 & 8 & 1 & 15 \\
\hline $\mathbf{N}$ & Barbados & 0 & 2 & 0 & 6 & 1 & 1 & 2 & 1 & 13 \\
\hline & Hungary & 0 & 1 & 0 & 2 & 0 & 2 & 1 & 2 & 7 \\
\hline $\mathbf{G}$ & Tchikoslovakia & 15 & 2 & 1 & 2 & 1 & 1 & 0 & 3 & 25 \\
\hline & Bermuda & 1 & 0 & 3 & 0 & 4 & 1 & 4 & 0 & 13 \\
\hline
\end{tabular}

54. The table is a compilation of data provided by the Jordanian Ministry of Industry and Trade. See http:/www.mit.gov.jo/mit/owa/k_sts2e (last visited Apr. 16, 2006). The information regarding 
The table above reveals that most of the foreign trademarks that are registered in Jordan originate in developed countries, namely the United States, The United Kingdom, France, Switzerland, Germany, Italy, The Netherlands and Japan. $^{55}$ Significantly, throughout the surveyed period, the number of trademark registrations originating in developed countries has been gradually increasing while the number of registrations by Jordanians, in Jordan, has been decreasing, this despite Jordan's amendment of its trademark law in accordance with TRIPS. Furthermore, on the relative level, the number of Jordanian marks registered locally does not exceed the total number of foreign marks registered in Jordan.

\subsection{The Concept of "Trademark Potential"}

In the first three sections of this chapter, I have examined the scope of trademark registration in Arab countries with respect to three elements, specifically: "Absolute," "Relative" and "Particular." In this section, I examine the extent to which Arab economies can use trademarks in the future. I refer to this as the "Trademark Potential" of Arab countries.

This concept of "Trademark Potential" submits that the ability to use trademarks in any country is inherently connected with the economic and commercial structure of that country. In this regard, a given country or region might participate in world trade within any of three sectors or a combination thereof, namely raw materials, manufactured products and services. Each of these has a different "Trademark Potential." "Trademark Potential" of a country (or region) it is necessary to consider the structure of its economy vis-à-vis these three economic sectors. But, before embarking on this task, it is first important to develop some understanding of the nature of these three economic sectors.

\footnotetext{
trademark registrations in Jordan is provided in the form of annual figures. The countries depicted in the table are categorized into three country groups namely: "Arab," "Developed" and "Developing."

55. Developing countries account for only a small portion of trademark registrations in Jordan. Similarly, most Arab countries account for a negligible amount of trademark registrations. Trademarks originating in Saudi Arabia, Syria, Kuwait and the U.A.E, have a notable share in trademark registration in Jordan but remain well below the number of trademark registrations originating in developed countries.

56. Lee E. Preston, Trade Patterns in the Middle East 7 (American Enterprise Institute for Public Policy Research 1970) (suggesting such a tri-sector distinction).
} 


\subsubsection{Raw Materials}

This trade sector involves raw materials that are extracted, mined or harvested from nature. Typically, this includes petroleum, natural gas, phosphates, minerals, wheat, corn, cotton, cacao beans, coffee beans, tea leafs, sugar canes and tobacco leaves. These "extractive" and "agricultural" commodities undergo processing that transforms them into products that are in demand by end-consumers including fuel, gas for cooking/heating, minerals, cereals, chocolate, coffee, tea, sugar, and tobacco. ${ }^{57}$

Typically, these raw materials are sold and shipped in bulk to the manufacturers or producers while they are in a "generic" state (i.e. coffee, tea, phosphates, metal ores, oil, cocoa, rice, etc.). As such, these primary products are said to have a very low "trademark potential." In essence, they are, by definition, in a "trademark barren" state. For example, tropical consumer goods such as coffee; cocoa beans, tea and tobacco leafs are traditional exports of developing countries and are consumed in massive quantities worldwide. However, developing countries do not enjoy trademark potential in such exports because these commodities are exported in bulk and in a raw state. In most cases, no (domestic) trademarks are used at this (shipment) stage. ${ }^{58}$

57. VIDA, supra note 18 , at 24 , referring to the Pearson Report that classifies primary and raw materials into four subgroups. These primary products include industrial raw materials including rubber; cotton and hard fibers. Also, included are mining products such as minerals; metals; phosphates; gas; oil and ores (copper, zinc, and lead).

58. These products, much like other primary goods, are shipped under their generic "identity" as simply "coffee," "tea," "cocoa," and "tobacco." It is only after undergoing processing and packaging that they assume their new "branded" identity. It is worth noting that some developing countries have managed to acquire distinct brands of their own, but in most of these rare cases the brand is only identified by bulk purchasers and does not appear on the final product. It is only at the end of the manufacturing process that these crude/raw materials are transformed into a product that is identifiable by the end-consumer. Furthermore, agricultural produce such as sugar, rice, cereals and meat are also exported in bulk and not under trademarks. More, surprisingly, fats, oils and dairy products (e.g. cheeses) are generally exported from developing countries without trademarks. This, despite their suitability for direct retail trade and their relatively high trademark potential. Indeed, in developed countries cheeses, oils, and dairy products are marketed and sold fewer than hundreds of different trademarks. See VIDA, supra note 18, at 32, also points out that some exceptional cases have involved goods trademarked by the country of export including SAMBA for edible oil from Brazil, MILAK (with woman head device) for peanuts and cashew nuts from India, MA-LING for peanuts from the Peoples Republic of China. 


\subsubsection{Manufactured Products}

This category covers a wide array of manufactured products directed towards the end consumer ranging from the very basic or mundane industry ("Basic Manufactured Products") to the very complex and high-tech products ("Advanced Manufactured Products"). While "Basic Manufactured Products" such as thread and plastics have a low "trademark potential," "Advanced Manufactured Products" have a very high "trademark potential" because virtually all of these products bear some kind of distinctive marking, rendering them the most "fertile" in terms of trademark use and registration. ${ }^{59}$

\subsubsection{Services}

The term "services" could be construed to cover a host of services ranging from the very basic such as human labor (e.g. workers in construction or agriculture) to the very complex such as medical services, accounting, investment, $R \& D$ and Internet services. While in the former type of services there is no use of marks (no trademark potential), in the latter type of services there is expansive use of marks (high trademark potential). In between those two ends are a multitude of services including, transportation, retail, communications, food, entertainment, security, etc. Table 10 depicts and summarizes the trademark potential of these three economic sectors.

59. Significantly, even in cases involving products with a high trademark potential such as textiles there are very few brands that originate in developing countries. This is because clothes are manufactured in developing countries, then shipped to markets worldwide while bearing trademarks owned by Western corporations. European companies like Marks\&Spencer and C\&A as well as leading footwear manufactures such as Nike, Puma, and Adidas are but a few that operate in such a manner. See VIDA, supra note 18, at 33. Notwithstanding, some developing countries have been selling various products including textile products under domestically owned trademarks. These countries include The People's Republic of China, India, Iran, Hong Kong, Taiwan and Israel. See VIDA, supra note 18, at 34, notes some examples of these brands including: DIAMOND (with a brilliant diamond figure); RED HORSE (with the design of two red horses); SOAR (with eagle design); MIMI (with cat design). All of these marks are used to sell clothing items. However, these cases remain the exception to the rule. 
Table 10

Summary of the "Trademark Potential" of Economic Sector Types

\begin{tabular}{|c|c|c|c|c|}
\hline $\begin{array}{l}\text { Economic } \\
\text { Sector Type }\end{array}$ & $\begin{array}{l}\text { Economic Sub- } \\
\text { Type }\end{array}$ & Goods/Services Contained in Type & $\begin{array}{l}\text { Identity of End- } \\
\text { Consumer }\end{array}$ & $\begin{array}{l}\text { "Trademark } \\
\text { Potential" }\end{array}$ \\
\hline Extractive & ----------- & $\begin{array}{l}\text { The natural resource endowment } \\
\text { of a country or region such as } \\
\text { raw materials, extracted, mined } \\
\text { or harvested ;including } \\
\text { petroleum; potassium; minerals; } \\
\text { metal ores; agricultural raw } \\
\text { products including cotton; rice; } \\
\text { coffee beans; cacao beans; tea } \\
\text { leafs; dried tobacco; natural gas; } \\
\text { coal; natural rubber. }\end{array}$ & Industry & Very Low \\
\hline \multirow[t]{2}{*}{ Manufacture } & $\begin{array}{l}\text { Basic } \\
\text { manufactured } \\
\text { products }\end{array}$ & $\begin{array}{l}\text { Simple consumer products or } \\
\text { initial low-grade processing of } \\
\text { raw materials (initial processing } \\
\text { of agricultural products; initial } \\
\text { refining of mined products such } \\
\text { a petroleum; coal; basic } \\
\text { consumer goods including - } \\
\text { plastics; basic products and } \\
\text { foods stuffs). }\end{array}$ & $\begin{array}{l}\text { Industry and } \\
\text { consumer } \\
\text { public }\end{array}$ & Low \\
\hline & $\begin{array}{l}\text { Advanced } \\
\text { manufactured } \\
\text { products }\end{array}$ & $\begin{array}{l}\text { High grade consum er goods } \\
\text { (including clothing; footwear; } \\
\text { apparel; medicines; } \\
\text { pharmaceuticals; computers; } \\
\text { cars; electronics; electric; } \\
\text { processed foods; toys; glasses; } \\
\text { watches; personal items; } \\
\text { furniture; cigarettes; beverages) }\end{array}$ & $\begin{array}{l}\text { Consumer } \\
\text { public }\end{array}$ & Very High \\
\hline \multirow[t]{2}{*}{ Services } & Basic services & $\begin{array}{l}\text { Essentially based on manpower } \\
\text { and human labor (including in } \\
\text { industry production lines; } \\
\text { factories; textile; agriculture; } \\
\text { construction; labor, drivers; } \\
\text { guides; and waiters. And also } \\
\text { including basic retail and food } \\
\text { services and private local } \\
\text { restaurants). }\end{array}$ & $\begin{array}{l}\text { Industry and } \\
\text { consumer } \\
\text { public }\end{array}$ & Very Low \\
\hline & $\begin{array}{l}\text { Advanced } \\
\text { services }\end{array}$ & $\begin{array}{l}\text { Sophisticated services requiring } \\
\text { a high degree of skill; capital; } \\
\text { organization; know-how } \\
\text { (including medical; accounting; } \\
\text { communications; media; } \\
\text { consulting; insurance; transport; } \\
\text { lodging; management; chain- } \\
\text { stores; restaurant chains; } \\
\text { brokerage; investment; law; } \\
\text { capital venture; research; } \\
\text { academics). }\end{array}$ & $\begin{array}{l}\text { Consumer } \\
\text { Public }\end{array}$ & High \\
\hline
\end{tabular}

According to research concluded in 1969, 90\% of foreign currency earnings of developing (non-industrialized) countries were composed of primary 
products. ${ }^{60}$ Furthermore, at that time, $50 \%$ of all developing countries based their entire export activity on a single primary commodity. This situation has remained largely unchanged because according to another research that was conducted three decades later, $80 \%$ of developing countries received $70 \%$ of their export earnings from raw materials such as minerals and unprocessed agricultural products. ${ }^{61}$

\subsection{Assessing the "Trademark Potential" of Arab Countries}

As alluded to above, there is a clear link between the "trademark potential" of a country and the commodities, products and services that comprise its economy. This section examines the "Trademark Potential" of Arab countries for a period covering three decades (1970-2000). This is done by taking into account commodities and services that are exported and/or imported by Arab countries, as well as by considering Arab countries' major trading partners. ${ }^{62}$

In 1964, Arab countries' exports were highly concentrated in a handful of commodities. During the 1960s, all non-oil-producing Arab countries have achieved a similar per-capita income, wherein agriculture commanded a substantial share in economic activity. ${ }^{63}$ Evidently, as early as 1964, and as demonstrated in Table 11 (below) the composition of Arab countries'

60. Collaborates in Development: Report of the Commission on International Development (C.B. Pearson ed., Praeger, 1969) concluding that $60 \%$ of developing countries earned $60 \%$ of the respective export receipts from three primary products. See also VIDA, supra note 18, at 24.

61. Beverly M. Carl, Trade and the Developing World in the 21st Century 19 (Transnational Publisher, Inc., Ardsley, New York 1998). Carl also notes that "technological developments have further reduced the demand for primary products of developing countries; for example, synthetic materials replacing cotton and wool. Industrial primary materials are exported (upon extraction) in large quantities, to industrialized countries (primarily the U.S., Western Europe and Japan) where they are subsequently processed (and refined) then sold under various trademarks to consumers worldwideincluding the same exporting countries. For example, crude oil extracted from the Middle East and other developing regions is subsequently refined then sold back to those developing countries under foreign (non-Arab) trademarks such as EXXON, SHELL, CHEVRON, and MOBIL. Even when the refining process takes place in the same country of extraction, foreign companies manage the process, and the end product is sold under foreign marks. See also VIDA, supra note 18, at 32.

62. This in order to establish a link between specific trading partners and specific goods, Preston, supra note 56 , at 15 .

63. Id. at 42. Preston notes that in 1964 the respective National product of Egypt, Jordan and Syria was (in millions of U.S. dollars) 4,120, 375 and 941 respectively. What is more, the Per-capita income for the same period was 151,194 and 172 U.S. dollars respectively. Agricultural exports constituted $26 \%$, $24.9 \%$ and $36.9 \%$ of total exports from those countries. 
economies reflects a low "trademark potential" because its exports are dominated by primary materials and agricultural produce.

Table 11

Export Specialization Among Arab Countries (1964) ${ }^{64}$

\begin{tabular}{|l|l|c|}
\hline \multicolumn{1}{|c|}{ Country } & \multicolumn{1}{c|}{$\begin{array}{c}\text { Type of } \\
\text { Commodities }\end{array}$} & $\begin{array}{c}\text { Percentage of } \\
\text { total exports }\end{array}$ \\
\hline Egypt & Cotton & 48 \\
\hline & Rice & 13 \\
\hline Iraq & Petroleum & 94 \\
\hline Jordan & Phosphates & 28 \\
\hline & Fruits and Vegetables & 23 \\
\hline Syria & Cotton & 51 \\
\hline & Wheat and Maize & 13 \\
\hline
\end{tabular}

This pattern has largely remained unchanged during the last four decades. Tables 12 and 13 below, detail the structure of imports and exports of selected countries for the period 1970-1986.

64. Id. at 43 (referring to Yacoub Mokhlis Zaki, The Impact of Arab Economic Integration on the Egyptian Economy (March 1969) (Unpublished Doctoral Dissertation), University of California, Berkeley). 
Table 12

Import Structure of Commodities by Main Categories (1970-1986) ${ }^{65}$

\begin{tabular}{|c|c|c|c|c|c|c|c|c|c|}
\hline \multirow{3}{*}{ Country } & \multicolumn{9}{|c|}{ By Main Categories of Imports (average percentage for the period 1970-1980) } \\
\hline & \multirow{2}{*}{$\begin{array}{l}\text { All food } \\
\text { items }\end{array}$} & \multirow{2}{*}{$\begin{array}{l}\text { Agricult- } \\
\text { ural raw } \\
\text { materials }\end{array}$} & \multirow[t]{2}{*}{ Fuels } & \multirow{2}{*}{$\begin{array}{l}\text { Ores and } \\
\text { metals }\end{array}$} & \multirow{2}{*}{$\begin{array}{l}\text { Manufa- } \\
\text { ctured } \\
\text { goods }\end{array}$} & \multicolumn{3}{|c|}{ of which } & \multirow{2}{*}{$\begin{array}{l}\text { Unalloc- } \\
\text { ated }\end{array}$} \\
\hline & & & & & & $\begin{array}{l}\text { Chemical } \\
\text { Products }\end{array}$ & $\begin{array}{l}\text { Other } \\
\text { manufac- } \\
\text { tured } \\
\text { goods }\end{array}$ & $\begin{array}{l}\text { Mach- } \\
\text { inery and } \\
\text { equip- } \\
\text { ment }\end{array}$ & \\
\hline Egypt & 28.8 & 5.8 & 5 & 8.7 & 51.6 & 10 & 15.2 & 26.3 & 0.2 \\
\hline S. Arabia & 18 & 1.4 & 0.7 & 6.8 & 72.9 & 4.4 & 29.3 & 39.3 & 0.2 \\
\hline Syria & 19.5 & 3.4 & 23.8 & 9.4 & 43.7 & 8.9 & 14 & 20.9 & 0.1 \\
\hline Jordan & 21.4 & 2 & 15.7 & 6.2 & 51.4 & 6.5 & 21.8 & 23.1 & 3.3 \\
\hline U.A.E. & 11.3 & 1.2 & 8.1 & 9 & 69.3 & 4.4 & 2.9 & 36 & 1 \\
\hline Tunisia & 17.2 & 3.9 & 11.4 & 9.5 & 57.4 & 8.4 & 21.3 & 27.7 & 0.7 \\
\hline Israel & 11.4 & 2.8 & 15.2 & 7.6 & 61.7 & 7.3 & 27.1 & 27.3 & 1.3 \\
\hline Turkey & 5.2 & 2.9 & 31 & 11.7 & 49.1 & 15 & 6.3 & 27.9 & 0 \\
\hline France & 11.9 & 4 & 20.7 & 9.1 & 54.2 & 9.4 & 20.4 & 24.3 & 0.2 \\
\hline U. States & 9.2 & 2.7 & 19.3 & 7.9 & 58.5 & 4.2 & 22.2 & 32.1 & 2.3 \\
\hline U. K. & 15.2 & 4.7 & 12.2 & 8.5 & 57.8 & 7.4 & 23.8 & 26.7 & 1.6 \\
\hline Singapore & 14.5 & 6.9 & 25 & 5.1 & 53.1 & 5.4 & 18.2 & 29.6 & 1.5 \\
\hline Japan & 15.1 & 8.8 & 40.3 & 12.2 & 22.7 & 5.7 & 8.6 & 8.5 & 0.9 \\
\hline Sweden & 7.7 & 2.2 & 17.7 & 9.3 & 62.7 & 9.2 & 22.5 & 31.1 & 0.4 \\
\hline Italy & 15.5 & 7.2 & 24.7 & 9.9 & 41.9 & 9.2 & 12.3 & 20.5 & 0.9 \\
\hline
\end{tabular}

Evidently, there is a clear fundamental difference between the import structures of developing countries and between the import structures of developed countries. While the former countries are heavily dependent on imports of manufactured goods, developed countries are largely dependent on fuels imports. In this regard, Saudi Arabia and Japan provide the most vivid example of this divergence. Indeed, while $72.9 \%$ of Saudi imports are comprised of manufactured products, fuels constitute $40.3 \%$ of Japan's total imports. The next table details the export structure of various countries for the period 1970-1986.

65. UNCTAD, Handbook of International Trade and Development Statistics, Supplement 1987, 163-78, U.N. Doc. (1988). The percentage figures appearing in this table constitute a simple average of data provided by the United Nations for the years: 1970, 1975, 1980, 1983, 1984, 1985 and 1986. The figures for the following countries, appearing in this table, are based on slightly different years: Saudi Arabia (1970, 1975, 1980, 1981, 1982); Syria (1970, 1975, 1980, 1982, 1983, 1984); U.A.E. (1970, 1975, 1979, 1980, 1981, 1982); Turkey $(1970,1975,1980,1983,1984,1985)$. 
Table 13

Export Structure of Commodities by Main Categories (1970-1986) (Average percentage of total commodity exports by country)

\begin{tabular}{|c|c|c|c|c|c|c|c|c|c|}
\hline \multirow[t]{3}{*}{ Country } & \multicolumn{9}{|c|}{ By Main Categories of Exports (average percentage for the period 1970-1986) } \\
\hline & \multirow{2}{*}{$\begin{array}{l}\text { All food } \\
\text { items }\end{array}$} & \multirow{2}{*}{$\begin{array}{l}\text { Agricult- } \\
\text { ural raw } \\
\text { materials }\end{array}$} & \multirow[t]{2}{*}{ Fuels } & \multirow{2}{*}{$\begin{array}{l}\text { Ores and } \\
\text { metals }\end{array}$} & \multirow{2}{*}{$\begin{array}{l}\text { Manufa- } \\
\text { ctured } \\
\text { goods }\end{array}$} & \multicolumn{3}{|c|}{ of which } & \multirow{2}{*}{$\begin{array}{l}\text { Unalloc- } \\
\text { ated }\end{array}$} \\
\hline & & & & & & $\begin{array}{l}\text { Chemical } \\
\text { Products }\end{array}$ & $\begin{array}{l}\text { Other } \\
\text { manufac- } \\
\text { tured } \\
\text { goods }\end{array}$ & $\begin{array}{l}\text { Mach- } \\
\text { inery and } \\
\text { equip- } \\
\text { ment }\end{array}$ & \\
\hline Egypt & 10.4 & 22.9 & 45.4 & 3.7 & 17.6 & 1.9 & 15.4 & 0.3 & 0 \\
\hline S. Arabia & 0.1 & 0 & 99.3 & 0 & 0.6 & 0.1 & 0.2 & 0.3 & 0 \\
\hline Syria & 9.2 & 16.6 & 62 & 1.5 & 10.5 & 1.2 & 8.5 & 0.9 & 0.1 \\
\hline Jordan & 27.6 & 0.7 & 0.1 & 37.7 & 33.8 & 15.7 & 16.8 & 1.4 & 0 \\
\hline U.A.E. & 0.7 & 0.1 & 97.2 & 0.3 & 1.5 & 0.1 & 0.8 & 0.7 & 0.2 \\
\hline Tunisia & 13.7 & 1.5 & 39.9 & 8 & 36.7 & 14.5 & 19.2 & 3.1 & 0.2 \\
\hline Israel & 13.6 & 3.8 & 0 & 3.2 & 79 & 13.8 & 50.7 & 14.5 & 0.4 \\
\hline Turkey & 40 & 14 & 3.2 & 10.5 & 32.3 & 2.6 & 25.9 & 3.7 & 0 \\
\hline France & 16.2 & 2.5 & 3.3 & 9.8 & 67.6 & 12.4 & 20.8 & 34.4 & 0.6 \\
\hline U. States & 16.4 & 4.6 & 4.3 & 4.9 & 66.4 & 9.9 & 12.2 & 44.3 & 3.5 \\
\hline U. K. & 7 & 1.6 & 13.8 & 6.7 & 68.3 & 11.6 & 21.4 & 35.4 & 2.5 \\
\hline Singapore & 9.4 & 10.3 & 26.2 & 3 & 45.4 & 4.9 & 12.8 & 27.7 & 5.7 \\
\hline Japan & 1.4 & 0.9 & 0.3 & 11.9 & 84.4 & 5.2 & 23.7 & 55.5 & 1 \\
\hline India & 30.5 & 4.4 & 3.5 & 11 & 50.4 & 3.6 & 39.6 & 7.1 & 0.2 \\
\hline Italy & 7.4 & 1.3 & 4.8 & 6.2 & 79.7 & 7.8 & 38.5 & 33.5 & 0.5 \\
\hline
\end{tabular}

The table above clearly indicates that the exports of Arab countries are dominated by fuels, crude petroleum and agricultural raw materials. Conversely, developed countries primarily export manufactured goods, two thirds of which are machinery and equipment. For example, fuels account for $99.3 \%, 97.2 \%, 62 \%$ and $45.4 \%$ of the exports of Saudi Arabia, U.A.E., Syria and Egypt respectively. On the other hand, manufactured goods account for $84.4 \%, 79.7 \%, 68.3 \%, 67.6 \%$ and $66.4 \%$ of exports from Japan, Italy, the U.K., France and the U.S. respectively.

What is more, the exports of Saudi Arabia, Syria, Egypt, Jordan, U.A.E., Tunisia, Kuwait and Iraq are dominated by primary raw materials such as crude petroleum, natural and manufactured gas, coal, etc. In contrast, the exports of France, Finland, Italy, Japan, the U.S., the U.K., South Korea and Switzerland are dominated by manufactured products.

Evidently, Arab countries have a limited export sector in terms of commodity types because their exports are based on a small number of commodities. Furthermore, Arab countries' exports (much like those of most developing countries) are based on commodities with a low trademark potential (e.g. crude petroleum, cotton, petroleum products, threads, cement, 
tea, coffee, fresh vegetables, fertilizers, etc.). In contrast, developed countries boast exports with a high trademark potential (e.g. road motor vehicles; telecommunications equipment; clothing; footwear; watches and clocks; alcoholic beverages; non-electrical machines; office machines; electrical machinery).

As reflected by tables 14, 15 and 16, (below) the structure of exports and imports of Arab countries has remained largely unchanged through 1985-1993. ${ }^{67}$ Here a distinction is made between two groups of Arab countries: those that are primarily dependent on oil production and those that have a more diversified economy. The first group includes the six countries of the Gulf Cooperation Council (G.C.C.) specifically Kuwait, Oman, Qatar, Saudi Arabia, U.A.E. and Bahrain. The other group, with more diversified economies, includes Egypt, Jordan, Syria and Yemen.

67. Economic and Social Commission for Western Asia: Analytical Review of Developments and Issues in the External Trade and Payments Situation of Countries of ESCWA Region, UniTED NATIONS app. 2 (1996) [hereinafter ESCWA]. 
Table 14

Overall Average Commodity Structure of Exports by Arab Countries (1985-1993)

(Percentage Share in the Commodity Sector)

\begin{tabular}{|c|c|c|c|c|c|c|c|c|}
\hline \multirow{2}{*}{\multicolumn{2}{|c|}{ Type of Commodity }} & \multicolumn{3}{|c|}{ Arab Countries (General) } & \multicolumn{4}{|c|}{ The Four Arab Countries Focused on } \\
\hline & & Total & GCC & $\begin{array}{l}\text { Diver- } \\
\text { sified }\end{array}$ & $\begin{array}{l}\text { Saudi } \\
\text { Arabia }\end{array}$ & Egypt & Jordan & Syria \\
\hline \multicolumn{2}{|c|}{$\begin{array}{l}\text { Food \& live } \\
\text { animals }\end{array}$} & 2.09 & 1.34 & 9.98 & 0.93 & 8.62 & 13.96 & 9.39 \\
\hline \multicolumn{2}{|c|}{$\begin{array}{l}\text { Beverages \& } \\
\text { tobacco }\end{array}$} & 0.19 & 0.18 & 0.38 & 0.049 & 0.13 & 0.75 & 0.35 \\
\hline \multicolumn{2}{|c|}{$\begin{array}{l}\text { Inedible crude } \\
\text { materials }\end{array}$} & 1.46 & 0.38 & 12.59 & 0.44 & 8.89 & 38.08 & 8.84 \\
\hline \multicolumn{2}{|c|}{ Mineral fuels } & 80.36 & 83.82 & 44.47 & 89.77 & 43.89 & 0.09 & 53.9 \\
\hline \multicolumn{2}{|c|}{$\begin{array}{l}\text { Animal \& } \\
\text { vegetable oils and } \\
\text { fats }\end{array}$} & 0.05 & 0.05 & 0.059 & 0.03 & 0.04 & 0.29 & 0.01 \\
\hline \multicolumn{2}{|c|}{$\begin{array}{l}\text { Total } \\
\text { manufactured } \\
\text { goods }\end{array}$} & 12.38 & 10.48 & 32.11 & 8.76 & 37.74 & 46.2 & 27.4 \\
\hline \multirow{4}{*}{$\begin{array}{l}\text { o } \\
\text { f } \\
\text { w } \\
\text { h } \\
\text { i } \\
\text { c } \\
\text { h }\end{array}$} & Chemicals & 4.08 & 3.75 & 7.55 & 6.19 & 2.98 & 27.5 & 7.45 \\
\hline & $\begin{array}{l}\text { Manufac- } \\
\text { tured } \\
\text { goods (by } \\
\text { materials) }\end{array}$ & 4.31 & 2.98 & 18.18 & 1.19 & 28.47 & 11.89 & 11.95 \\
\hline & $\begin{array}{l}\text { Machinery } \\
\text { \& transport } \\
\text { equipment }\end{array}$ & 2.4 & 2.57 & 0.62 & 1.14 & 0.57 & 1.6 & 0.49 \\
\hline & $\begin{array}{l}\text { Miscellan- } \\
\text { ous } \\
\text { manufactur- } \\
\text { ed articles }\end{array}$ & 1.36 & 1.17 & 5.77 & 0.25 & 5.73 & 5.18 & 7.56 \\
\hline \multicolumn{2}{|c|}{ Unallocated } & 3.46 & 3.75 & 0.41 & 0.08 & 0.75 & 0.91 & 0.03 \\
\hline
\end{tabular}


Table 15

Overall Average Commodity Structure of Imports by Arab Countries (1985-1993)

(Percentage Share of Various Commodity Sectors)

\begin{tabular}{|c|c|c|c|c|c|c|c|c|}
\hline \multirow{2}{*}{\multicolumn{2}{|c|}{ Type of Commodity }} & \multicolumn{3}{|c|}{ Arab Countries (General) } & \multicolumn{4}{|c|}{ The Four Arab Countries Focused on } \\
\hline & & Total & GCC & $\begin{array}{l}\text { Diver- } \\
\text { sified }\end{array}$ & $\begin{array}{l}\text { Saudi } \\
\text { Arabia }\end{array}$ & Egypt & Jordan & Syria \\
\hline \multicolumn{2}{|c|}{$\begin{array}{l}\text { Food \& live } \\
\text { animals }\end{array}$} & 15.22 & 12.7 & 22.72 & 12.45 & 23.62 & 24.76 & 19.06 \\
\hline \multicolumn{2}{|c|}{$\begin{array}{l}\text { Beverages \& } \\
\text { tobacco }\end{array}$} & 1.25 & 1.3 & 1.15 & 1.09 & 1.51 & 0.59 & 0.14 \\
\hline \multicolumn{2}{|c|}{$\begin{array}{l}\text { Inedible crude } \\
\text { materials }\end{array}$} & 2.72 & 1.77 & 5.53 & 1.79 & 7.89 & 3.05 & 2.78 \\
\hline \multicolumn{2}{|c|}{ Mineral fuels } & 4.88 & 3.99 & 7.28 & 0.28 & 2.54 & 16.12 & 10.64 \\
\hline \multicolumn{2}{|c|}{$\begin{array}{l}\text { Animal \& } \\
\text { vegetable oils and } \\
\text { fats }\end{array}$} & 0.89 & 0.4 & 2.26 & 0.39 & 2.67 & 1.19 & 1.81 \\
\hline \multicolumn{2}{|c|}{$\begin{array}{l}\text { Total } \\
\text { manufactured } \\
\text { goods }\end{array}$} & 71.72 & 75.72 & 59.71 & 77.03 & 60.58 & 57.22 & 64.63 \\
\hline \multirow{4}{*}{$\begin{array}{l}\mathbf{o} \\
\mathbf{f} \\
\mathbf{w} \\
\mathbf{h} \\
\mathbf{i} \\
\mathrm{c} \\
\mathrm{h}\end{array}$} & Chemicals & 7.99 & 7.16 & 10.57 & 6.71 & 10.96 & 10.09 & 12.78 \\
\hline & $\begin{array}{l}\text { Manufac- } \\
\text { tured } \\
\text { goods (by } \\
\text { materials) }\end{array}$ & 20.66 & 20.69 & 20.7 & 23.39 & 20.31 & 18.12 & 24.86 \\
\hline & $\begin{array}{l}\text { Machinery } \\
\text { \& transport } \\
\text { equipment }\end{array}$ & 31.67 & 34 & 24.12 & 32.93 & 25.91 & 21.35 & 24.47 \\
\hline & $\begin{array}{l}\text { Miscellan- } \\
\text { ous } \\
\text { manufactur- } \\
\text { ed articles }\end{array}$ & 11.4 & 13.87 & 4.33 & 16.5 & 3.39 & 7.69 & 2.52 \\
\hline \multicolumn{2}{|c|}{ Unallocated } & 3.34 & 4.08 & 1.48 & 0.34 & 1.2 & 3.77 & 0.94 \\
\hline
\end{tabular}

Tables 14 and 15 further affirm that while Arab exports are primarily based on (crude) mineral fuels, Arab imports are based, largely, on manufactured products. $^{68}$

68. Research indicates that the share of manufactured exports from the Middle East and North Africa regions (MENA region) is on the rise. According to on source, manufactured exports from the MENA region have risen from $10.7 \%$ in 1990 , to $16.4 \%$ in 1995 , with an annual growth rate of 12.6 percent. However, despite this rise, that research observes that "dynamic manufactured products remain under-represented in the region's exports, their proportion out of the total manufactured output is still negligible, and their diversification beyond traditional markets is modest." MENA, Macroeconomic Performance and Global Integration: Trends and Forecasts, Chapter 1: Overview of MENA, Macroeconomic Performance, http://www.erf.org/html/body-Chap1.html (last visited Mar. 15, 2007). 
From all of the above it is possible to conclude that while Arab countries' exports are primarily comprised of mineral fuels and inedible crude materials, their imports are dominated by manufactured goods. Consequently, while their export sector has a very low trademark potential, their import sector is dominated by products with a high trademark potential. ${ }^{69}$

The clearest indication of the low trademark potential of Arab economies is demonstrated by its massive oil sector which, in 1993, produced a staggering 4.2 billion barrels of oil. In 1998, over $27 \%$ of the world's oil was produced by Saudi Arabia, Kuwait, Iran, Bahrain, Iraq, Qatar, and the U.A.E. with a combined production totaling 17.1 million barrels a day. In that same year Saudi Arabia exported 7.9 million barrels (of oil) a day that accounted for $46 \%$ to total Gulf oil exports for that year. Furthermore, these countries are thought to possess $64 \%$ of the world's oil reserves. ${ }^{70}$

In 2000, mining products accounted for $96.6 \%$ of the Middle East's exports of primary products, and for $74.7 \%$ of all of its merchandise exports. Thus, it is not surprising that Arab economies have become synonymous with oil extraction and imports. ${ }^{71}$ This issue is not only connected with the

69. In this regard, it is also worth noting that food and live animal imports have decreased in importance during this period 1985-1993. According to the ESCWA, supra note 67, at 17, this decline is attributed to the "agricultural promotion policies undertaken in most countries of the region." See Antoine B. Zahlan, Middle East Executive Reports 274 (indicating that Arab Countries are importing advanced civilian technologies). See also James J. Emery et al., Technology Trade With the Middle East 89-116 (Westview Press 1986).

70. See ESCWA, supra note 67, at 22-23 (referencing OPEC (Organization of Petroleum Exporting Countries Statistical Bulletin and quoting information provided by the U.S. Energy Information Administration (EIA)). See also Business Indicators, 22 Middle East Executive Reports, No. 4, 23 (Apr. 1999) (stating that the annual oil exports of these countries for the period 1985-1993 show that Saudi Arabia and the U.A.E. are the largest two oil-exporting countries in the region while Syria is the smallest oil exporter. Iraq's and Kuwait's oil exports in 1991 were slashed due to the Iraqi invasion of Kuwait. Oil exports in Kuwait recovered while Iraqi exports did not due to UN-imposed sanctions.).

71. See Saudi Arabia: Planning Beyond Current Conditions, SABIC Moves Ahead on Petrochemicals, 22 Middle East Executive Reports, No. 2, 8 (Feb. 1999) the Saudi Arabia Basic Industries Corporation (SABIC) initiated four expansion projects (worth about 3 million US\$) including construction of new ethylene glycol; chemical grade method; ammonia; urea; and ethylene facilities. Oil production in Saudi Arabia has always been on the rise. In 1999 (despite the fact that 1998 was a year of depressed oil prices), SABIC has moved ahead in increasing annual production of petrochemicals (at its 15 plants). Oil production rose even during 1998 (when oil prices slumped) by 5\%. Furthermore, the volume of marketed products rose $7 \%$. These down stream petrochemical products include aromatics and PVC-Based products. Except for Africa, no such region even nears these figures. Furthermore, in developed regions, the situation is almost the complete opposite; mining products account for only a modest (7.2\%) share of total merchandise exports of North America, Western Europe and Asia. In 2000, mining products accounted for only $8.8 \%$ of all goods imported into the Middle East. The price of oil has a direct effect on the credit quality of banks in the Arabian Gulf. In the wake of the 1998 decline in oil prices, those commentators contended that "prolonged low oil prices present a direct threat to the profitability, liquidity 
trademark potential of Arab countries but also has other implications. Indeed, the World Bank contends that the economic stagnation of the region should be blamed on the dependence of Arab countries on oil. However, analyzing the reason for this dependence is beyond the scope of this research. ${ }^{72}$

In conclusion, while most of the output of Arab countries is based on products with a low trademark potential, most imports into Arab countries are composed of manufactured products with a high trademark potential.

and ultimately, the solvency of Gulf banks." Indeed, not only is the banking system affected by the low oil prices, the entire economies of the Gulf countries including the privet sector are dependent on the sale of oil and its derivative ("downstream") products. Not surprisingly then, that oil not only dominates exports but also constitutes the "lion share" of GDP in Gulf countries members of the Gulf Cooperation Council (GCC)). See Andrew Cunningham \& Adel Satel, The Outlook for Banks In Light of Low Oil Prices, 21 Middle East Executive Reports, No. 8, 13 (Feb. 1999). Similarly, crude oil accounts to $60 \%$ of Syria's total exports and one third of its GDP; see Henry T. Azzam, The Region: Outlook for Growth in 2000, 22 Middle East Executive Reports No. 11, 9, 18 (Nov. 1999). Indeed, oil forms the backbone of Arab industry and is the dominant component of its export structure. In this regard, one research observes that the West Asia region "participates in world trade activities, but it is highly concentrated in terms of commodity composition and direction. Oil and oil products are the dominant exports of the region, while imports are mainly manufactured goods largely obtained from developed market economies." See ESCWA, supra note 67 , at 3 .

72. See www.worldbank.org (search "world development indicators 2002 " follow hyperlink "News \& Broadcast-World Development Indicators 2002") (The World Bank contends that

the Middle East and North Africa region has been unable to achieve sustained growth. Saudi Arabia, the largest economy in the region, has grown about $0.8 \%$ a year since the 1960 s. Egypt's economy has been growing at an average rate of $3.2 \%$ a year for the past 40 years, helped by large aid transfers. But 26 years after the first oil boom, the region's economic fortunes are still driven by international oil prices.

The World Bank, 2002 World Development Indicators, at 195. For the text of the World Bank, U.S. World Development Report, see http://www.cl.bas.bg/libraries_BAS/cl.html; http://www.worldbank.org (visited Oct. 2, 2002). Other, even more, "bold" research establishes a link between major world events of the last century and the natural wealth of oil embedded in the ground of the Middle East). See generally WILLIAM Engdal, A Century of War: Anglo-American Oil Politics and the New World Order (Pluto Press 2004) (1992) that research submits that the oil reserves (the "Black Gold") in the Middle East are the main reason for the two World Wars and the GulfWar of 1991, as well as other regional and international events. Two factors may help explain why Arab countries depend on oil: Firstly: It is only logical (and even detrimental) that a given country would opt to exploit its natural endowments. Thus even when Arab countries have attempted to diversify their economy and to shift away from petroleum in the direction of industry, they have only done so within the natural extension of oil extraction and oil related production. Therefore, "since their [Arab Countries] endowments are in oil and natural gas, establishing industries in these fields should not surprise anyone." See Public Enterprises and Development in Arab Countries-Legal and MANAGerial 14 (International Center for Law Development 1977) (In this regard, it is worth mentioning that ever since the early 1970s, Arab economies have been expanding their activity into oil-related industries. Specifically, 35.4\% of public enterprises in Arab Gulf states were linked to oil and natural gas industries.). 
Therefore, no change in those countries" "trademark balance" can be expected while their exports are based on products with a low "trademark potential." 73

In contrast, developed countries manifest a reverse trend by shifting from exports with a low trademark potential to exports of manufactured products that generally have a high trademark potential. Thus, not only is the average GDP of developed countries (Western Europe, the U.S. and Japan) about 10 times that of the entire Middle East and North Africa region, the composition of their respective exports varies greatly. According to the World Bank, developed countries have, since the 1970 s, been increasing their merchandise exports and decreasing the export of primary commodities. Specifically, in 1995 exports of manufactures from Germany, Japan and the United States respectively constituted about $90 \%, 96 \%$ and $82 \%$ of their overall exports. ${ }^{74}$ Table 16, below, summarizes the composition of merchandise trade according to product for the period 1963-2000 for the three leading industrial countries/regions (the U.S., Western Europe and Japan).

73. The dominance of oil is further deduced from the fact that Arab countries that are not major oil exporters have a very low volume of exports. In fact, Saudi Arabia, the highest ranking merchandise exporter among Arab countries, is only number 20 on the list well, below the major industrialized countries and other countries like Belgium, Sweden and Singapore. In addition to Saudi Arabia, other Arab countries on the list of top 50 merchandise exporting countries including U.A.E., Algeria, Kuwait, Iraq, all of which are major exporters of oil. Evidently, oil and related merchandise is the only type of merchandise that is exported (by Arab countries) in substantial quantities in the context of international trade. Non-oilproducing Arab countries such as Egypt, Morocco, Jordan, Syria, and Lebanon do not appear on the list; i.e. that their annual merchandise exports are below those of Vietnam (US \$14.5 billion). See WTO, International Trade Statistics, 2001, at 23.

74. See generally Michael Czinkota, Ilkka Roukainen \& Michael Moffett, International Business Update 14 (5th ed., The Dryden Press 2000) (referring to statistics by the World Bank, World Development Report 1996. From Plan to Market, Rep. No. 15892 (June 1996), available at www.wds.worldbank.org/ external/default/WDSContentServer/WDSP/IB/1996/06/27/000009265_3961214181445/Rendered/PDF/ multi0page.pdf). 
Table 16

Merchandise Trade of North America, Western Europe and Japan

${\text { (By Product })^{75}}^{75}$

(1963-2000)

(Average Percentage)

\begin{tabular}{|l|l|l|l|l|l|l|}
\hline \multirow{2}{*}{ Merchandise } & \multicolumn{3}{|c|}{ Exports } & \multicolumn{3}{c|}{ Imports } \\
\cline { 2 - 7 } & U.S.A.* & $\begin{array}{l}\text { West } \\
\text { Europe }\end{array}$ & Japan & U.S.A.* & $\begin{array}{l}\text { West } \\
\text { Europe }\end{array}$ & Japan \\
\hline Total Merchandize & 100.0 & 100.0 & 100.0 & 100.0 & 100.0 & 100.0 \\
\hline Agricultural Products & 20.4 & 13.6 & 3.3 & 13.8 & 18.8 & 28.4 \\
\hline Food & 14.7 & 10.6 & 2.1 & 10.1 & 13.8 & 16.7 \\
\hline Raw Materials & 5.7 & 3 & 1.3 & 3.7 & 5 & 11.8 \\
\hline Mining Products & 9.8 & 8.6 & 1.4 & 16.7 & 16.9 & 35.8 \\
\hline Ores \& other Minerals & 2.6 & 1.1 & 0.1 & 2.1 & 2.2 & 7.4 \\
\hline Fuels & 4.9 & 5.3 & 0.3 & 12 & 12 & 25.5 \\
\hline Non-Ferrous Metals & 2.3 & 2.2 & 0.9 & 2.6 & 2.7 & 3 \\
\hline Manufactures & 65 & 75.5 & 93.7 & 66.4 & 62 & 34.4 \\
\hline Iron \& Steel & 1.4 & 4.7 & 8.6 & 2.7 & 3.5 & 1.1 \\
\hline Chemicals & 8 & 11 & 5.8 & 4.9 & 8.7 & 6 \\
\hline Other Semi-Manufactures & 6.4 & 9.5 & 6.7 & 7.8 & 7.8 & 3.2 \\
\hline $\begin{array}{l}\text { Machinery and Transport } \\
\text { Equipment }\end{array}$ & 40.4 & 35.2 & 56.9 & 36.3 & 28.3 & 14.4 \\
\hline Power Generating Machinery & 1.5 & 1.1 & 0.9 & 0.8 & 0.9 & 0.8 \\
\hline Other Non-Electrical Machinery & 8.8 & 10.6 & 9.8 & 6 & 6.9 & 3.1 \\
\hline Office and Telecom Equipment & 9.1 & 5.7 & 16.8 & 9.7 & 6.8 & 5.8 \\
\hline $\begin{array}{l}\text { Electrical Machinery and } \\
\text { Apparatus }\end{array}$ & 3.4 & 4.2 & 5.6 & 3.2 & 3.5 & 1.9 \\
\hline Automotive Products & 11.3 & 9.6 & 15.4 & 14.2 & 7.2 & 1.4 \\
\hline Other Transport Equipment & 6.2 & 4 & 8.4 & 2.5 & 3 & 1.6 \\
\hline Textiles & 1.3 & 4 & 6.1 & 2.2 & 3.3 & 1.5 \\
\hline Clothing & 0.6 & 2.4 & 1.1 & 3.6 & 2.9 & 2.6 \\
\hline Other Consumer Goods & 6.7 & 8.4 & 8.5 & 8.9 & 7.4 & 5.4 \\
\hline
\end{tabular}

* The term "North America" in the table includes the U.S.A. and Canada.

75. The numbers in the above table are the resultant of a simple average of the data published by the WTO for the years 1963, 1973, 1983, 1993, and 2000. See generally WTO, International Trade Statistics, supra note 73, at 31-33. 
Table 16 reveals that a large share of the exports of these developed regions is composed of office and telecommunications equipment; power generating machines; automotive products; clothing and other consumer goods. These products typically possess a very high trademark potential. On the other hand, food, raw materials, ores and other minerals and textiles have a very low share in exports. Thus, it is possible to conclude that, exports of North America, West Europe and Japan are typically of a high trademark potential (i.e. manufactured products and machinery). Furthermore, these industrialized countries are the primary consumers of Gulf oil. For example, in 1998, oil from the Gulf region accounted for $75 \%$ of oil imports to Japan. In fact, $31 \%$ of Japan's oil imports from the Middle East region are from Saudi Arabia and $34 \%$ are from the U.A.E. ${ }^{76}$ Furthermore, Gulf oil constituted $21.9 \%$ and $50 \%$ of total imports of oil to the U.S. and Western Europe respectively. ${ }^{77}$

76. WTO, International Trade Statistics, supra note 73 , at 23 ; noting that $25 \%$ of oil came from Iran and about $19 \%$ came from Iraq. Japan's oil imports from the Gulf remained relatively unchanged from 1997 ( 75 percent) because of a significant drop in Japan's oil demand ( 0.2 million b/d). Indicators provided by the World Bank support the findings as to the dominance of petroleum in the exports of Arab countries and other developing countries. This is reflected by the World Bank as a "negative" import. According to the World Bank, countries that display such a "negative import of oil include Saudi Arabia, Egypt, Syria, Kuwait, Algeria, Yemen, Tunisia, Nigeria, Angola, Turkmenistan, Mexico, Iran, Malaysia, Venezuela, Norway, the United Kingdom and Canada. See World Bank, World Development Report 2000-2001, Table 10, Energy Use and Emissions 2000-2001, at 292-93. An additional indication regarding the industrial activity of countries is reflected in the volume of commercial use of oil (both in absolute values and as a per capita ratio). Oil is used to power engines and to produce electricity for factories, transportation, and shipping. The U.S. has the largest volume of commercial energy use as well as the highest per-capita use of oil. China is the country with the second highest level of commercial use (not of per capita energy use!). Russia is ranked third after China. Other countries that use massive amounts of oil for commercial use are Japan, India, Germany, the U.K., France, South Korea, Brazil and Mexico. Not surprisingly, those countries produce the highest emissions of carbon dioxide. Interestingly, although Saudi Arabia is the largest exporter of oil in the world ( $92 \%$ of its exports), its commercial energy use is comparable with that of Poland and is equivalent to only $30 \%$ of Germany's commercial energy use. Furthermore, Egypt's commercial energy use is less than that of Belgium. Furthermore, the interconnection between oil and Middle Eastern exports was demonstrated during 1998 Asian Economic Crises wherein Arab exports were reduced due to a sharp drop in demand for oil by Asian economies. In that year, Asian imports (mainly oil) from the Middle East declined by about $17 \%$ and Middle Eastern and African exports fell by about $22 \%$ and $16 \%$ respectively. WTO, International Trade Statistics 2001, Value of World Merchandise Trade by Region (1993-2000), www.wto.org (visited June 24, 2006), at 37. Another commentator discussing the effect of the 1997 Asian economic crises on Arab countries, points to the link between the decline in Arab economies and the fall of oil prices whereby "[the Asian economic crises] resulted in a weakening of global demand for oil, which has led to the spectacular decline in oil prices since October 1997. This decline will have major implications for balances of payments and underlying capital flows in many Arab countries." Also see Paul Chabrier, Arab Financial Systems: Lessons from Asia and Future Challenges, MiddLE EAST EXecutive Reports, vol. 21, No. 8, Aug. 1998.

77. See WTO, International Statistics, supra note 73 (noting that the U.S. oil imports from the Gulf region hit a 25-year low of 31 million b/d in 1985). In 1990, oil imports rose to $1.97 \mathrm{million} \mathrm{b} / \mathrm{d}$. and then 
An additional indication of the low trademark potential of Arab countries is reflected by the countries that are their major trading partners. Indeed, if those trading partners trade manufactured products for raw materials, then this would provide another indication of the low trademark potential of Arab countries.

Countries of Western Europe, the U.S. and Japan have been the major trading partners of Arab countries for a period covering the last four decades. ${ }^{78}$ Indeed, even as early as 1966 the share of imports from Western Europe, the U.S. and Japan dominated the Middle East market (70.3\% of all imports). Similarly, $72.5 \%$ of Middle East exports were directed to those developed countries. In contrast, during that period, Arab intra-regional trade constituted a very small portion of trade by Arab countries to be precise, $6.5 \%$ of exports and $8.5 \%$ of imports.

During 1970-1986 a major share of Arab exports to Japan, Europe and North America was dominated by oil-producing Arab countries. On the other hand, exports by non-oil-producing Arab countries were not directed towards developed markets. In other words, where Arab oil exports end up in industrialized countries, other Arab exports did not manifest the same clear trend. For example, while 65\% of Saudi Arabia's exports were directed to developed countries, the bulk of Jordan's exports (83.6\%) ended up in developing countries. What is more, the bulk of Arab imports for that period (1970-1986) were from developed countries. Notably, despite the creation of the WTO at the end of 1994, no change is manifested in these trade patterns. ${ }^{79}$

From the previous (five) tables above, it is possible to deduce an important characteristic of Arab trade that has a bearing on the trademark potential of Arab countries. Suffice it to note that in the last four decades the European Union, Japan and the United States were the prime destination for Arab exports (over 55\% of exports from Arab countries) and were the source

declined gradually through 1996, until increasing again in 1997-1998; U.S. oil imports in 1977-1998 reached 2.45 and 2.22 million b/d respectively in 1998, nearly $16 \%$ of U.S. oil imports came from Iraq and $14 \%$ from Kuwait and very small amounts from Qatar and the U.A.E. The United States imported in that year 2.14 million $b / d$ of oil from the region; amounts equivalent to those of 1977-1978. Business Indicators, supra note 70, at 23 . What is more, $70 \%$ of the United States oil imports (from the Gulf) came from Saudi Arabia. Similarly, 48\% of West Europe's oil imports for the Gulf (in 1998) came from Saudi Arabia. Much like the United States, Western Europe's oil demand from the region rose significantly from $22 \%$ in 1996 to $27 \%$ in 1998. Japan's imports of oil from the region in 1998 constituted the majority of its net oil imports.

78. Preston, supra note 56, at 22-23; United Nations, Trade Statistics, §§ 3.4-3.5 (1988).

79. ESCWA, supra note 67, at 23; International Monetary Fund (IMF), Direction of Trade Statistics Yearbook, 1993 and 1994, Washington D.C.; United Nations, Trade Statistics 1988, New York-Geneva, Section 3.5, at 125-33; see Azzam, infra note 117, at 15. 
of $61 \%$ of imports by Arab countries. Furthermore, intra-Arab trade constitutes only $9 \%$ of all Arab trade, which is much lower than the level of intra-regional trade in other world regions. ${ }^{80}$ The structure of Arab foreign trade and the low intra-Arab trade is largely influenced by the type of raw materials that are traded and mainly oil, the predominant export commodity for many Arab countries. ${ }^{81}$ Indeed, during the period 1958-1966, oil constituted about $50 \%$ of intra-regional trade in the Middle East. In other words, Arab countries conduct almost no trade with each other because most of the products that they trade in are primarily raw materials namely extractive (oil), which, have a very low trademark potential. ${ }^{82}$

A further indication of the low trademark potential of Arab countries may also be deduced from the trade patterns between the U.S. and Arab countries. The U.S. has a (slight) negative trade balance with oil-exporting Arab countries (such as Saudi Arabia, Kuwait and Algeria) and a very high trade surplus with the non-oil-exporting countries (such as Egypt, Jordan, Syria and Lebanon). ${ }^{83}$ Thus, in effect, non-oil-exporting countries (that export products with low trademark potential) have very little to export to the U.S. while oilexporting countries have a trade surplus with the U.S. due to their oil exports. $^{84}$ This imbalance is also evident in other sectors including franchising, services and super-brands.

80. Interestingly, the figures of inter-Arab trade for the years 1996 and 1999 remain almost unchanged; this despite the Arab Free Trade Agreement of 1997. In 2000, inter-Arab trade stood at only $8.6 \%$ of total trade. In comparison, intra-regional trade in the European Union, East Asia and the North American Free Trade Area (NAFTA) was 60\%, 40\% and 37\% respectively. See Azzam, infra note 117.

81. ESCWA, supra note 67 (stating that even Egypt and Syria had expanded their own oil production until their oil production superseded their national demand).

82. PReston, supra note 56, at 38-39 (citing 1957-1966 UN Stat. Y.B. Int'1 Trade) (predicting, in 1970, that Egypt (The United Arab Republic at the time) and Syria would themselves become net exporters of oil)).

83. Business Indicators, U.S. Trade with Near East/North African Countries (1992-1994), 18 Middle East Executive Reports, No. 3, at 31 (Mar. 1995); U.S. Department of Commerce, Office of the Near East, Feb. 17, 1995; Business Indicators: U.S. Trade with Near East/North African Countries (1996-October 1998), 21 Middle East Executive Reports at 22 (Sept. 1998). U.S. Department of Commerce, Office of the Near East; Business Indicators: U.S. Trade with Near East/North African Countries (1997-2000), 23 Middle East Executive Reports, No. 3 at 20, 12, Dec. 2000, at 20.

84. See the 1998 Country Reports on Economic Policy and Trade Practices: Near East, U.S. States Department Publication, http://www.state.gov/www/issues/economic/trade_reports/neareast98/index.html. This source details the trade balance (in millions of dollars) of some Arab countries with the U.S. for the year 1998: Algeria (953); Jordan (-287.2); Kuwait (70); Morocco (-336); Tunisia (-310); Oman (-53.8); Saudi Arabia (1,200); Syria (-140); U.A.E. $(-1,400)$; Egypt data pertaining to the year $1997(-3,146)$. 


\subsubsection{Franchising ${ }^{85}$}

Western brands boast a major share in the franchising activity in Arab countries. For example, in Saudi Arabia franchising has proved to be "especially appealing" to entrepreneurs and consumers. At the end of the year 2000 , investment in franchising, in that country, totaled US \$250 million with a staggering annual growth rate of $27 \%$. Notably, fast food franchises stood out by controlling an estimated $35-40 \%$ of the entire franchising market in the Kingdom. Franchising in Saudi Arabia is dominated by U.S. corporations, which at the beginning of 2001 accounted for $60-70 \%$ of that market. European companies dominate the other 30-40\%. American and European corporations operate in the Saudi franchise market through brands such as: Guess; Anne Klein; Berlitz; GNC; MailBoxes etc.; Budget; Hertz; Avis; McDonald's; Benetton; BHS; Zara and Mango. ${ }^{86}$ These corporations have encountered (almost) no local or regional competition. Furthermore, there is a growing interest among other U.S. brand owners for franchising in Saudi Arabia including Victoria's Secret, The Gap, The Limited, Banana Republic, U-Haul, Olive Garden, Red Lobster and Gymboree. ${ }^{87}$ The expansion of foreign franchising in Saudi Arabia is typical of other Arab countries. In this regard, one commentary observes that:

Franchising has found fertile ground in the Middle East in the past decade and is expected to become increasingly popular throughout the region. Solid consumer demand, the popularity of foreign products, and the attractiveness of the franchising

85. WIPO, supra note 48, at 66 (stating that franchising is practiced in a host of business sectors including automobiles, home appliances, prepared foods, beverages, rental of capital equipment (e.g. automobiles, trucks), hotel operations, dry cleaning facilities or even secretarial help. The sale of goods or the rendering of services under the franchise is usually based on a trademark, service mark (or trade name) or even a special design or décor of the business establishment. The license of these marks by the "franchiser" is typically coupled with the supply (by the franchiser) or know-how and/or management services. Thus, the main benefit to the franchisee stems from the "reduction of the risks associated with establishing and starting up a business." Upon setting up the outlet, the franchisee has at his disposal an experienced entity; the franchiser. In addition, he need not build recognition for his brands since it already exists among local consumers. The concept of franchising has proven to be an ingenious model for expanding the use of successful brands (trademarks and/or service marks). Broadly defined, a franchise (or distributorship) is "a business arrangement whereby the reputation, technical information and expertise of one party are combined with the investment of another party for the purpose of selling goods or rendering services directly to the consumer.").

86. Saudi Arabia's Franchising Market: Relatively Untapped and Lucrative, 23 MidDLE EAST Executive Reports, No. 12, Dec. 2000, at 13-14. The report suggests that "the reason for the success of U.S. franchises in Saudi Arabia is because "many young Saudis have lived and studied in the U.S. and are familiar with the customer service, decor and efficiency these franchises offer." (on file with author).

87. Id. 
formula have lead to the creation of hundreds of new franchises in Arab countries. Major U.S. franchises - Burger King, McDonald's, Pizza Hut, Avis, Hertz, Hilton, Holiday Inn, to name a few - are well represented throughout the region. Indeed, as consumer demand continues to grow, there is room for more restaurant/hospitality, quick printing, dry cleaning, retail and convenience, and other franchising outlets. ${ }^{88}$

Arab consumers, much like other consumers worldwide, are becoming increasingly exposed to foreign brands through various channels of advertising, the Internet and travel overseas. It is not surprising that Arab markets with the absence of local brands (low trademark potential) have become increasingly fertile ground for foreign brands.

Another sector of Western franchising in the Middle East involves the hotel services sector in post-war (1982) Lebanon. The table below details the most prominent of these foreign hotel brands.

\section{Table 17}

\section{Hotel Groups Operating in Post (1980s) War Lebanon ${ }^{89}$}

\begin{tabular}{|l|l|l|}
\hline \multicolumn{1}{|c|}{ Hotel Name } & \multicolumn{1}{|c|}{ Year Opened \& Location } & \multicolumn{1}{c|}{ Hotel Brand/Group } \\
\hline Marriott & 1996 & MARRIOTT \\
\hline Vendome Intercontinental & 2000 & INTERCONTINENTAL \\
\hline Phoenicia Intercontinental & 2000 & TNTERCONTINENTAL \\
\hline Faraya's Mzaar Intercontinental & 2000 & TNTERCONTINENTAL \\
\hline Holiday Inn Martinez & Beirut & HOLIDAY INN \\
\hline Holiday Inn Dunes & Beirut & HOLIDAY INN \\
\hline Crown Plaza & Hamra, Beirut, 2001 & CROWN PLAZA \\
\hline Novotel & Various locations in Lebanon & ACCOR-NOVOTEL \\
\hline Sofitel & Various locations in Lebanon & ACCOR-NOVOTEL \\
\hline Mercure & Various locations in Lebanon & ACCOR-NOVOTEL \\
\hline Sheraton & 3 Major Locations & STARWOOD-SHERATON \\
\hline Hilton & Limited Presence in Service & HILTON \\
\hline Rotana-Gefinor & Apartments in Beirut & \\
\hline Quality Inn & Clemenceua-Beirut & ROTANA HOTELS \& RESORTS \\
\hline Comfort Savoy & City of Tripoli & CHOICE HOTELS \\
\hline Versaille & Beirut & CHOICE HOTELS \\
\hline Comfort/Howard John son & Hamra-Beirut & HOWARD JOHNSON \\
\hline Best Western & Hazmeih-Beirut & HOWARD JOHNSON \\
\hline & 2 Hotels in Beirut & BEST WESTERN \\
\hline
\end{tabular}

In view of the low trademark potential of Arab countries it should come as no surprise that most franchising activity in Arab countries involves foreign

88. Jamil Zouaoui, Quick Guide to Negotiating and Establishing Franchises in the Region-with Examples from a Kuwaiti Experience, 21 Middle EAst Executive Reports, No. 9, Sept. 1998, at 9 (on file with author).

89. Bahij Abi Ghanem, Franchising an Industry, Middle East Travel, (Sept./Oct. 2001), 23, at 24 (on file with author). 
brands. Consequently, the marketing power enjoyed by foreign brands allows the foreign franchiser to tailor the franchising agreement according to his needs.

Most of the above mentioned hotel groups franchise their brands in what are referred to as "tight franchises" whereby the franchiser dictates strict conditions pertaining to building specifications, interior design and type of equipment, this in order to secure a "unified identity" for all hotels operating under a specific brand. Furthermore, this type of franchising allows the franchiser to be involved in managing the hotel, namely by appointing his delegates among the top managerial positions in the hotel. Additionally, the franchiser receives various types of monetary compensation including franchise fees, revenue fees and shares in net profits. ${ }^{90}$ These payments reduce the profits of the local franchisee, thus, the franchisee's home country collects less taxes. ${ }^{91}$

It is worth noting that foreign hotel groups (operating in Lebanon) do not finance projects or inject capital into the Lebanese economy. Thus, it could be argued that local hotel brands could have generated the same employment and business opportunities. ${ }^{92}$ In light of this, it could be argued that, aside from their promotional value, foreign hotel brands do not boost the local economy, but serve their own business interests. ${ }^{93}$

90. Id. at 25; detailing these fees: A. Franchise fee: a onetime fee generally determined by the number of rooms in the hotel; B. Revenue fees: typically about $3 \%$ of revenues (constituted as $1 \%$ for administration, $1 \%$ for the use of the service mark and $1 \%$ as a form of reservation fee); C. Share in net profits from running the hotel; this ranges between 8 to 17 percent. In another type of franchises (referred to as "loose franchising") the brand owner does not get closely involved in the running management of the business. However, even in such franchises certain services are rendered by the franchiser who also maintains a certain degree of control. What is more, franchisees pay fees to the franchiser for use of the brand and the services rendered by the latter.

91. Zouaoui, supra note 88 , at 9 . The franchise agreement binds the local franchisee; he is required to provide personal guarantees to the franchiser, and he is required to share in both the business management and the revenues of his business. What is more, a franchisee does not own the brand that he is operating under. Therefore, he can achieve neither long term planning nor expansion (i.e. establishing a new franchise business of his own).

92. In effect, the local franchisee bears the investment costs that are connected with setting up the hotel business (through local investments or bank loans). As such, Ghanem, supra note 89, concludes that the only benefit brought about by foreign brands is "limited to providing an indispensable service of marketing and promotion through channels that lead to the globalization of member institutions."

93. In this context, Ghanem, supra note 89 , at 25 is adamant that "it is about time a Lebanese hotel brand which embodies the country's identity was launched . . it is about time the idea of franchising a Lebanesebrand name became a reality." This position has some merit when considering tourism in pre-war Lebanon, the Lebanese cuisine and the success of Lebanese entrepreneurs in launching and boosting tourism and hotel businesses in the Arab world (and particularly in the Gulf region). However, in view of the already established presence of foreign hotel brands in Lebanon, this challenge does not promise to be 


\subsubsection{Services}

Half a century ago, the world's leading economies were predominantly production oriented. Since then, trade in services has become a substantial source of income for the world's leading economies and continues to grow rapidly worldwide. ${ }^{94}$ One commentary submits that growth in the service sector is because "many formally non-traded services are now being newly traded." 95 Consumers' demand for a variety of services is also growing and becoming increasingly more sophisticated. In this regard, it has been suggested that "recent advances in information technology have transformed the service sector from a collection of non-tradable products with a low productivity growth potential to a sector comprising a variety of fast-growing, knowledge-based products such as banking, insurance, and technical services. $" 96$

Today, a wide variety of services are being offered including finance; insurance; communications; transport; freight; consultation; research; investment; advertising; distribution; education; medical. The increasing importance of services has brought about its integration into the multilateral trading system under the WTO-GATT framework. ${ }^{97}$ Consequently, the use of service marks has become increasingly widespread.

The TRIPS agreement, which provisions are now incorporated into the laws of most Arab countries, relates to trademarks and service marks in equal

\footnotetext{
an easy one. Indeed, it appears that brands that are owned and managed by multi-national corporations (conglomerates) hamper the competitive ability of local industry. This applies not only to hotel services but also to a host of services and retail sectors. For example, fast-food chains and book stores are but a few visible examples of such domination. A vocal critique of the power vested (through brands) in the hands of MNCs is Neomi Klien. See N. KLIEN, No Logo (St. Martin Press 2002).

94. Jaime Serra et al., Reflections on Regionalism: Report of the Study Group on International Trade, CARnegie Endowment for Internation AL Peace, Washington D.C., 1997, at 35-36 submits that exports of services more than doubled between 1980 and 1993 from US $\$ 760$ billion to US $\$ 1,881.7$ billion and "now accounts for almost one quarter of its total trade and is growing faster than merchandise trade." Between 1980 and 1993 the average annual growth in world trade in commercial services was 7.7 percent; whereas the corresponding rate for merchandise was only $4.9 \%$. ESCWA, supra note 67 , at 7. Furthermore, the share of services within worldwide commercial activity rose from $17 \%$ in 1980 to about $22 \%$ in 1993 . See MEDA, Chapter 3.3, http://www.erf.eg/html/body_chap33.html, at 2 (last visited Apr. 28, 2006). Serra et al., supra, at 36 (based on World Bank data (1995)).

95. For example, telecommunications and information technologies are becoming less costly and more easily distributed worldwide. ESCWA, supra note 67, at 7.

96. MENA, Chapter 3.3, http://www.erf.eg/html/body_chap33.html at 2 (visited May 27, 2006).

97. This has been achieved through the general Agreement on trade in Services (GATS).
} 
measure. ${ }^{98}$ Therefore, when examining the effects of trademark laws on Arab countries, it is important to also consider service marks. Indeed, the concept of "trademark potential" also covers service marks and distinguishes between services with a low "service mark potential" and services with a high "service mark potential." In order to determine the service mark potential of Arab countries it is necessary to consider two factors: the share of Arab countries in trade in services and the type of services being offered by Arab economies.

According to the World Trade Organization, the bulk of trade in services was controlled (in 1992) by industrialized and developed countries that are party to the OECD. These countries include (in order of volume of trade in services) the U.S., France, Italy, Germany, U.K., Japan, Spain, Holland, Belgium, Luxembourg, Austria and Australia. ${ }^{99}$ In 2000, Egypt and Saudi Arabia respectively ranked 30th and 40th on a list of the top 40 countries that are engaged in exporting services, and which together account for $92 \%$ of world trade in services. It is worth noting that the service exports of Egypt and Saudi Arabia (combined) accounted for only 1\% of world trade in services. ${ }^{100}$ In contrast, the U.S., U.K., France, Germany and Japan together account for $42.4 \%$ of world trade in commercial services. Curiously no other

98. TRIPS agreement (article 15(1)). In 1999, the "Nice Agreement Concerning the International Registration of Goods and Services for the Purpose of the Registration of Marks" was amended. Services originally classified under class 42 were re-categorized into four new classes (42-45).

99. Serra et al., supra note 94, at 36. Based on World Bank data (1995): OECD countries accounted for $81 \%$ of volume of service traded worldwide in 1993 . The Organization for Economic Cooperation and Development (OECD) is an organization which aim is to promote the following (set out in article 1 of the Convention signed on 14th December, 1960, and which came into force on 30th September, 1961):

to achieve the highest sustainable economic growth and employment and a rising standard of living in member countries, while maintaining financial stability, and thus to contribute to the development of the world economy; to contribute to sound economic expansion in member as well as non-member countries in the process of economic development; and to contribute to the expansion of world trade on a multilateral, non-discriminatory basis in accordance with international obligations.

The original member countries of the OECD are Austria, Canada, Denmark, France, Germany, Greece, Iceland, Ireland, Italy, Luxembourg, the Netherlands, Norway, Portugal, Spain, Sweden, Switzerland, Turkey, the United Kingdom and the United States. Other countries subsequently joined the OECD: Japan (1964), Finland (1969), Australia(1971), New Zealand (1973), Mexico(1994), the Czech Republic (1995), Hungary (1996), Poland (1996), and South Korea (1996). For economic indicators pertaining to OECD member states see OECD Report, Industrial Competitiveness: Benchmarking Business Environments in the Global Economy (OECD, Head of Publication Services, Paris France, 1997). Also see Fadi Ali Mathna, The Expected Effect of the WTO on External Trade and Developing Countries 65 (Madbouli Press, Cairo 2000).

100. WTO, International Trade Statistics, supra note 73, at 21. 
Arab countries are on this list, which shows that they only have a negligible share in world trade in services.

In Egypt, which is a non-oil-exporting country, services constitute 66\% of total exports. Indeed, Egypt and Jordan, hold a very high world ranking in the Revealed Comparative Advantage (RCA) in commercial services. ${ }^{101}$ The RCA of Egypt and Jordan, with respect to exports of commercial services, is much higher than the corresponding RCA of Japan, the U.K., Germany, South Korea and Italy. However, the service sector in Egypt and Jordan is dominated by the (government) public sector with only a marginal contribution to export capacities of these countries. ${ }^{102}$ In other words, despite Egypt's impressive RCA, its service sector has a low "service mark potential" because the type of services that contribute to this RCA are associated with the Suez Canal and remittance of Egyptian workers abroad, mostly to other Arab countries for work in the construction and agricultural sectors. Similarly, Jordan's high RCA is attributed to the remittance of migrant workers (to Arab countries). ${ }^{103}$ Furthermore, Egypt's tourism service sector holds a high RCA. However, as in Lebanon, "foreign firms manage most of the four and five star hotels in the country." 104 The domestic Egyptian tourism

101. The RCA of a country in the service sector is the ratio of the services in that country's exports to its share in world trade. RCA that is higher than one is indicative of that country's "relative specialization" in that sector. See MENA, supra note 68, at 3.

102. Preston, supra note 56, at 42. The government service sectors in Egypt have expanded by $100 \%$ between the late 1930 s and the early 1960 s. Preston observes that

the substantial and growing importance of the service sector-including domestic trade, government, education and so forth — within these economies [of the Middle East] has had a profound impact on the potentiality for expanded trade among them. With minor exceptions, the production of services is essentially a domestic activity; hence, there is virtually no possibility that increases in total and per capita product based upon increased service industry output will yield additional merchandise for export. On the contrary, increased activity within the service sector is more likely to increase domestic demand both for domestically produced goods and for imports. Id. at 43 .

103. MENA, supra note 68 , at 3 . Greece and Spain also have a high RCA that is largely due to tourism and remittance of migrant workers.

104. Going to and Fro: Egyptian Tourism; Inbound/Outbound, MiddleEast Executive Reports, vol. 23, No. 12, Dec. 2000, at 9. The situation is clearly exemplified in the case of Egypt. In 2000, 5.5 million tourists visited Egypt. With this surge, the hotel industry has flourished thus attracting foreign companies. The report lists some of the foreign and local hotel management companies active in the mark et such as: Sheraton Management; Marriott; Sonesta Nile Cruises; Conrad International; Holiday Inn; Hilton International; Intercontinental; Meridian Novotel; Movenpick. The foreign-owned management companies originate in the U.K., U.S., France, Switzerland, Denmark, and India. Much as in the case of Lebanon, substantial chunks of revenues revert to those brand-owning entities. On the other hand, it is important to recognize the quality services that are offered under those foreign-owned brands and their contribution to hotel services and tourism. See Ghanem, supra note 89, at 25. 
sector is composed of services with a low service mark potential including drivers, waiters and hotel staff. It does not have services with a high trademark potential such as hotel consulting and management. Another service sector that is prevalent in Arab countries is "transit" which includes not only "movement of vessels through the Suez Canal but also the flow of oil through pipelines and the storage and transportation of merchandise at major ports." 105 All of these have a very low trademark potential as well. Indeed, the high share of service exports in total exports and the high RCA of Egypt, Jordan and other Arab countries are not indicative of a high service mark potential. Similarly, Saudi Arabia's inclusion in the 40th place of major exporters is due to services associated with Muslim pilgrimage activities and oil industry related services.

Thus, despite the dominance of the service sector in the exports of Egypt and Jordan (as well as other Arab countries) their service mark potential remains low because of the types of services being offered.

\subsubsection{Super Brands}

In the hierarchy of trademarks, there stands out a select group of marks referred to as well-known marks that enjoy special renown and are accorded special protection even if not registered. ${ }^{106}$ Above this group of marks is an even more elite class of brands referred to as "Super Brands." Such brands are no longer only well-known or famous, but have become "cultural icons" in their own right. Marks such as NIKE, MICROSOFT, CALVIN KLEIN, ROLEX and SWATCH are examples of "super-marks that have become an integral part of consumers' psyche - and even vocabulary - through enormous advertising campaigns and positive media associations."107

105. Preston, supra note 56, at 10. A small number of government-controlled or private companies render these services. The services they provide do not necessitate the use of service marks. What is more, in the context of remittance of labor, no service mark potential can be established.

106. TRIPS Agreement, Jan. 1, 1995, arts. 16(1), 16(2). See also Tshimanga Kongolo, Are WellKnown Marks, Well-Known in African Countries?, 5 J. World Intell. Prop. 273, 273 (2000). Kongolo questions whether these well-known marks are well-known in African countries. This issue does not seem relevant in Arab countries in view of their openness to Western products and, the structure of their imports and economies. Id. at 286-89.

107. Alisa D. Lewis, The United States' Trademarks Century in Review: Brand Modernization and the Rise of the Mega-Brand-1960-2000, at 3, http://www.inta.org/index.php?option=com_content\&task= view $\&$ Itemid $=51 \&$ getcontent $=1 \& \mathrm{id}=200$ (last visited May 4, 2006). Super brands came of age in the second half of the last century. In that post world-war era, brands have played an increasing role in trade and marketing. These campaigns are boosted by the ever deepening penetration of television, radio, press, motion pictures, and the Internet whereby at "nearly every point during the day and evening, brand owners 
These marks have transcended their basic role (of identifying source) and have became indicators of social status and tools for cultural expression; they acquired the power to create wants and began to "shape and affect cultural trends." Consequently, the value of super brands has skyrocketed and exceeded the annual GDP of most countries on the globe. Indeed, the revenues of multi-national corporations that own these brands exceeds the GDP of most countries. The status of these marks has been enhanced by the legal protection accorded in TRIPS. In view of this, it is not surprising that the number of super brands has risen "exponentially."108

In 2002, U.S. corporations owned most (65\%) of the top 100 global brands. The remaining brands were owned by either European or Japanese corporations. ${ }^{109}$ Significantly, none of those leading brands originate in Arab countries or other developing countries for that matter. In other words, the most geographically far-reaching and powerful brands worldwide originate in developed countries, that are also referred to as countries of the "North." This is not surprising in view of the "trademark deficit" and the low "trademark potential" of Arab countries. Furthermore, most of those leading brands are owned by Multinational Corporations (MNCs) that enjoy a strong presence internationally and command extensive economic power. ${ }^{110}$

had the access to a captive and receptive consumer audience."

108. $I d$.

109. See BusinessWeek Online, 2002 Global Brand Scoreboard, http://bwnt.businessweek.com/ brand/2002/index.asp (last visited Nov. 10, 2005). See also Fortune.com, The Top 500 Global Corporations, http://www.fortune.com (last visited Nov. 13, 2005).

110. Multinational Corporations (MNCs) are networks of affiliated units located in various countries worldwide and headed by parent firms with a distinct nationality. The parent firm typically exercises some control over the dispersed corporations and formulates a common strategy. Despite the structure of these corporations and lack of a simple hierarchy, each MNC has a distinct nationality at its core. In addition to the vast volume of sales that MNCs enjoy, the larger MNCs generally operate in over 100 countries. What is more, the largest $500 \mathrm{MNCs}$ command $80 \%$ of the world's indirect investment and ownership of foreign affiliates. Furthermore, in some countries MNCs' affiliates account for more than one third of the output of the marketing sector. For example, IBM has operations in over 130 countries worldwide. See Czinkota, Ronkainen \& Moffett, supra note 74, at 396; the source of this table is the InTERNATIONAL 500, Forbes Magazine (July 28, 1997), (visited Sept. 13, 2006), at 218, also see Fortune, The Global 500, (Aug. 4, 1997), at 2. Also see http://www.forbes.com and http://www.pathfinder.com/fortune/ (visited Nov. 14, 2005). Kavaljit Singh, Global Corporate Power: Emerging Trends and Issues, Asia PAC. RESEARCH NETwORK, June 2001, at 3 (noting that many MNCs (or “Transnational Corporations") could be traced back to the "major colonizing and imperialist countries of Western Europe notably England and Holland" and citing the British East India Trading Company as a prime example). Multinational Corporations are not a new phenomenon. They have evolved through history, but have only recently (in the last four decades) expanded their reach and enhanced their status. Singh, supra, at 5 (noting that in the year 2000, the top 100 economies of the world were 54 MNCs and 46 countries, contrasted with 51 and 49 , respectively, in 1989). This is a further testimony to the growing power and influence of MNCs. 
In view of this power and the wide scope of merchandise and services that they produce, it is not surprising that MNCs own a large portion of trademarks worldwide. ${ }^{111}$

Even as early as the 1960 s, MNCs have been closely associated with developed-industrialized countries, specifically the United States (55\%), the United Kingdom (20\%), countries of Western Europe and Japan (12\% each). ${ }^{112}$ This situation has largely remained unchanged during the past four decades. The largest Industrial corporations worldwide for the year 1996 originate in the U.S., Japan, the U.K., France, Germany, and the Netherlands. ${ }^{113}$ Furthermore, in 1997, the corporate headquarters of the largest $500 \mathrm{MNCs}$ were located in only 32 countries including North America, Europe and the Far East. Furthermore, the top 100 MNCs originate in the U.S., Japan, Germany and France wherein the share of those countries is 37, 22,10 , and $7 \%$ respectively. ${ }^{114}$ Strikingly, none of the leading MNCs originated in Arab countries.

\section{Chapter Three \\ THE “M.A.R.T.I.N.A.” SCALE}

In view of the different factors that have been considered in the previous chapter, there is a need for a model that factors in all of these factors and produces an objective scale that can provide a clear indication as to the effects of trademark law on a given country. This chapter does just that.

111. See Are multinationals too powerful? What can be done about their power?, http:// www.angelfire.com/sc/iressays/Multinationals.html(last visited Aug. 3, 2007). For example, IBMhas been dubbed the "Patent King" because it registers (on average) 10 new patents each day (in the U.S. alone!). See Nicholas Varchaver, The Patent King, Fortune MAGAZINE, May 14, 2001, at 203; according to Singh, supra note $110,90 \%$ of all cross-border licensing payments and $70 \%$ of all international patent royalty payments are made between MNCs and their subsidiaries.

112. NAVIN JOSHI, THIRd WORLD, at 90.

113. Czinkota, Ronkainen \& Moffett, supra note 74, at 396; the source of this table is the International 500, Forbes Magazine, July 28, 1997) (visited Sept. 13, 2006), at 218, also see Fortune, The Global 500, Aug. 4, 1997, at 2. Also see http://www.forbes.com and http:// www.pathfinder.com/fortune/ (visited Nov. 14, 2005).

114. In fact, Arab countries have no share in MNCs even in the oil sector. Suffice it to note that oil production by Exxon Mobil exceeds oil production of all OPEC countries combined. Singh, supra note 110, at 5. Moreover, of the top $500 \mathrm{MNCs}, 185$ corporations are American and 104 corporations are Japanese. 


\subsection{Defining the M.A.R.T.I.N.A. Scale}

My proposed model incorporates a scale which I refer to as "The Matrix of Absolute and Relative Trademarking, Industrial and Negotiating Advantage" ("M.A.R.T.I.N.A."). It comprises three basic factors, namely "Trademarking" activity, "Industrial" orientation and "Negotiating" ability. The M.A.R.T.I.N.A. scale is depicted in the following formula:

Table 18

The "M.A.R.T.I.N.A." Scale and its Primary Factors

0 < M.A.R.T.I.N.A.” = Trademarking + Industrial + Negotiating $<50$

(15 Points) (Points 25) (10 Points)

The "Trademarking" Factor considers the Absolute and Relative levels of trademark registration. The Absolute level and the Relative level are awarded up to five points and up to ten points respectively. For example, countries with a high number of registered marks that are mostly registered by residents of that country would receive a high score in this factor.

The "Industrial" Factor focuses on the "trademark potential" and considers the relative size of the sector with a high "trademark potential" as compared with the entire economy of that country. For example, a country would be awarded up to 25 points if it reaches a "trademark potential" of $100 \%$ (i.e. if all of its national economy produces only products with a very high "trademark potential"). In contrast, countries like Saudi Arabia would be awarded a very low score because, as demonstrated above, the bulk of its production is oil (and oil extracts) that has a negligible "trademark potential." The "Negotiating" Factor considers the influence that a given country has had on the formulation of standards of trademark protection. Three issues are considered within this factor:

1. Does the country belong to a strong/influential regional group (i.e. EU, NAFTA, ASEAN, OPEC, G-8)? (up to 3 points);

2. Did the country play an influential role in the formulation of TRIPSWTO norms? (up to 3 points);

3. Does the country command substantial political authority that allows it to initiate effective negotiations pertaining to intellectual property issues (or to block such an attempt)? (up to 4 points).

The three factors of trademarking, industry and negotiating are awarded 15, 25, and 10 points respectively. Thus, according to the "M.A.R.T.I.N.A." 
scale, the maximum score for a country is 50 . Countries with a very high score (above 45 points) are expected to resist any change to the current trademark régime because such a régime would be considered beneficial to their economies. Conversely, countries that score below 15 points are least likely to benefit from stringent trademark protection. In order to clarify how this proposed scale functions, it is applied, below, to three countries, namely: Japan, Israel and Jordan.

\subsubsection{Japan}

During 1994-2000 an annual average of 153,780 trademarks were registered in Japan. The overwhelming majority of these registrations were by resident entities $(137,222)$ while only 16,558 trademarks were registered annually during that period by non-residents. Thus, on the absolute level, Japan would be awarded the full five points, while on the relative level it would receive 8.2 points. Thus, the total for the trademarking factor in the case of Japan would be 13.2 out of 15 points.

The "Industrial" Factor is also very high in the case of Japan because $80 \%$ of all Japanese exports are constituted of manufactured products including electrical and mechanical equipment and other consumer goods that have a high trademark potential. Consequently, Japan is awarded 21 points out of the full 25.

Lastly, in the "Negotiating" Factor, Japan receives six points because of its membership in the influential G-8 group and the TRIPS "Quad" that it took part in the negotiations leading up to TRIPS (three points for each). In addition, Japan is awarded three points (out of four) for its strong international standing today. In total, Japan receives 9 out of the 10 points in this factor.

The sum of all of these factors is 43.8 out of the maximum 50 points on the M.A.R.T.I.N.A. scale $(13.8+21+9)$. This high score indicates that Japan's economy is benefiting from high standards of trademark protection.

\subsubsection{Israel}

During the seven-year period of 1994-2000, on annual average, 6,379 trademarks were registered in Israel. On average only 1,479 of those were registered by Israeli residents, while 4,900 were registered annually by foreigners. Thus, on the "absolute" level, Israel would be awarded only two out of five points, and on the "relative" level Israel would be awarded only 1 point out of ten because the number of non-resident registrations exceeds that of locals. Consequently, Israel receives three points out of 15 for this factor. 
Israel fares better in the second factor, that of "Industry." Here the figures indicate that the Israeli economy is very vibrant because about $60 \%$ of all Israeli exports enjoy a high trademark potential. Therefore, this factor is awarded 15 points out of the maximum 25 points. The "Negotiating" factor indicates a mixed pattern. While Israel did not play a role in the negotiations leading up to TRIPS, and does not belong to any formal regional or international trading block, it does enjoy good economic and political standing, especially in view of its alliance with the West and mainly the U.S. (with which it has a free trade agreement). Thus, Israel is awarded 1 point (out of three for its membership in trading blocks), zero points for not playing an active part in formulating TRIPS and 3 points for its political and economic standing internationally.

In total, Israel gets 22 points (out of the maximum 50) on the "M.A.R.T.I.N.A." scale. This relatively low score indicates that Israel may wish to reconsider some of the standards of protection pertaining to trademarks that have been incorporated into its national legislation or to think about ways in which to increase awareness and utilization of the trademark law within its national economy. ${ }^{115}$

\subsubsection{Jordan}

On average 2,283 trademarks were annually registered in Jordan during 1994-2000. Of those, 1,329 were by non-residents and only 954 were by residents. Consequently, Jordan is awarded only one point (out of five) for the "absolute" level and one point (out of 10) for the "relative" level. Thus, in total, the "Trademarking" Factor is awarded only two points out the maximum 15 points.

Less than a third of Jordanian products as well as exports have any "trademark potential." The bulk of Jordanian exports are based on products and services with a low trademark potential. Therefore, for the "Industrial" factor Jordan is awarded only seven points out of 25 . In the "Negotiating" factor, Jordan is accorded only one point out of a maximum of three for its membership in a regional group (Arab Free Trade Area) because this regional group is very weak in comparison with the EU, NAFTA, ASEAN or the G-8. Furthermore, Jordan did not participate in formulating the trademark standards

115. Issues of parallel imports and expansive protection to foreign well-known marks may also be reconsidered. Furthermore, Israel may also examine ways in which to raise its score on the "M.A.R.T.I.N.A." scale namely by encouraging more registrations by locals and by raising the trademark potential of its economy. 
of protection as set out in TRIPS. Thus, no points are given for this. Lastly, Jordan's international standing has never been very high. It does have good connections with the West and the U.S., but it is doubtful whether this translates into any real influence towards reshaping intellectual property standards of protection. Therefore, Jordan is given only two points out of four. In total, the "negotiating" factor yields three points (out of the maximum ten) for Jordan.

Consequently, Jordan's score on the "M.A.R.T.I.N.A." scale is only 11 points out of fifty. This very low score indicates that Jordan is not, and most likely does not directly benefit from its modern law. The same rationale applies to other Arab countries because they are operating under very similar economic and political conditions, as demonstrated in Chapter Two of this research. It is worth noting that Saudi Arabia's score on the "M.A.R.T.I.N.A." scale is lower than that of Jordan's because Saudi production is completely dominated by products with a low trademark potential. Indeed, Gulf countries would be awarded even less points for this factor, because the bulk of their production and exports is based on oil and oil related products. Similarly, Syria's industry type and its very low international standing will award it a very low score on the "M.A.R.T.I.N.A." scale as well.

\section{CHAPTER Four \\ Assessments And Conclusions}

World trade in both goods and services has been growing and expanding. In 1998 global trade in goods amounted to a staggering US \$6.5 trillion dollars, and reportedly created 1.5 million new jobs. Likewise, trade in service has expanded, and in 1996 amounted to US $\$ 1.2$ trillion. ${ }^{116}$ Consequently, this expansion has contributed to the creation of new trademarks and service marks. But while trade and the registration and protection of marks has been expanding, very little information has been provided regarding the actual effect of trademark laws on countries. Indeed, literature has not provided any model for assessing and measuring the effects of trademark laws on countries.

In this research, I have introduced a model for measuring the effects of the trademark régime in any given country. I have applied this model to four Arab countries that are distinctly different from one another. In this regard, 
I have compiled and considered data pertaining to trademark registration in Arab countries. I have also considered the "trademark potential" of Arab countries through examining the structure of Arab economies and their trade patterns. In addition, I have focused on distinct sectors namely: services, franchising and super-brands. From the findings in this research, it is possible to conclude the following:

a. Arab countries lag behind developed countries in all three levels of trademark registration namely the "Absolute," "Relative" and "Particular." Arab countries are at a disadvantage in terms of actual trademark registration both within their respective jurisdictions and beyond. Not only is the number of registrations much smaller than that of developed countries, but, also the relative share of nonresident owned marks that are registered in Arab countries is much higher than the comparable rate in developed countries. Furthermore, the "particular level" indicates that developed countries dominate "foreign registrations" in Arab countries. This situation has been largely constant throughout three decades (1970-2000).

b. The "trademark potential" of Arab countries does not fare any better. I have introduced this concept in order to help predict the scope of trademark use in Arab countries in the future. Indeed, products and services that dominate Arab economies have a very low "trademark potential."117 Thus, despite the strong legal protection that is accorded by Arab countries to marks, the number of locally owned

117. Indeed, in 1999, the total exports from Arab countries, including petroleum exports, totaled a mere USD 163 billion less than the exports from Hong Kong that amounted to USD 174 billion in the same year. In that year, oil and minerals accounted for USD 118 billion of all exports by Arab countries. Thus, the volume of all other exports from Arab countries totaled only US $\$ 45$ billion that is less than the exports of Finland, a country of 5.5 million inhabitants. See Henry Azzam, Arab Countries Economic Cooperation: Changing Rhetoric to Reality, Middle East Executive Reports, vol. 24, No. 1, Jan. 2001, at 15. This limited export capability of Arab countries, has led some commentators to call for greater regional cooperation among Arab countries, especially, in view of the formation of regional trading blocks worldwide. For example, Azzam at 14 notes that "More serious cooperation is required if the Arab countries are to deal successfully with the critical challenges they face today-including weak economic growth, high unemployment rate, high internal and external dept levels, and limited export capabilities." My data analysis suggests that the trademark "deficit" of Arab countries is symptomatic of their economic stagnation and their overall "trade deficit." Indeed, the issues of external trade and national economic development appear to be intertwined. In 1978, a United Nations report submitted that in order to achieve economic developments for developing countries it is necessary to expand export earnings and to reduce import costs. See United Nations Conference on Trade and Development, Trade and Development Board, Tenth Special Session, Geneva, 19 Mar. 1997, Item 2 of the Provisional Agenda, Report on the Ad Hoc Group of Experts on the External Trade of the Least Developed Countries, Held at the Palais des Nations, Geneva, from 11 to 19 , Dec. 1978, at 5 . 
trademarks is not expected to rise. Indeed, Arab countries are not likely to increase the use of trademarks regardless of the strong protection that is accorded to marks by their national laws. Furthermore, the nature of the franchising activity within Arab countries and the bleak picture pertaining to super-brands and MultiNational Corporations (MNCs) provides additional support to this conclusion.

These findings show that while "Western" countries enjoy a robust manufacture-oriented economy with a trademark surplus and a high "trademark potential," Arab economies are predominantly extractive economies with exports dominated by oil and other primary products. ${ }^{118}$ This economic pattern, in Arab countries, has remained largely constant during the last four decades. Consequently, it appears unlikely that Arab countries will be able to raise their trademark potential and ultimately their trademark balance given their economic structure. In short, analysis of relevant data indicates that despite the modern trademark laws in Arab countries, they suffer from an acute "trademark deficit" and have a very low "trademark potential." Evidently, the trademark régime that has been adopted into the trademark laws of Arab countries has failed to generate a change within the economic structure of these countries and, in effect, its function has been primarily limited to protecting brands that are mostly foreign-owned and that dominate trademark registration therein.

These findings are in line with the views of "Dependency" theorists who argue against the blind importation of "Western" legal norms. Indeed, it appears as though imitating the formalistic legal structures of trademark protection erected by the West does not necessarily contribute towards improving the trademark balance of Arab countries and raising their low trademark potential. On the contrary, it appears that these laws only serve to encourage the entry of additional foreign brands into Arab markets without any notable "traffic" in the opposite direction. ${ }^{119}$ Indeed, there is a need to

118. In complete contrast to the situation in Arab countries, the U.S. economy has a mighty "trademark balance" and a very high trademark potential. American corporations own thousands of trademarks that cover goods or services that are demanded by the U.S. market as well as other international markets. James Gerber, International Economics, Addison Wesley, Reading, Massachusetts, 1999, at 4, contends that "the vast majority of goods and services we [Americans] consume are made at home. Haircuts, restaurant meals, gardens, healthcare, education, financial services, utilities and most of our entertainment, to name a few, are domestic products. In fact, about 87 percent of what we consume is made in the United States, since imports are equal to about 13 percent of our gross domestic product (GDP)."

119. Endeshaw, supra note 11, at 6-7, notes that

the advocacy for, and support of, borrowing by non-ICs is done in disregard of considerations that 
consider ways in which to remedy this trademark imbalance, however, that task is beyond the scope of this research.

may show certain IP forms as being more suitable for a certain country or time than for another country or for a different time. Much of the borrowing or formulation of IP policies and laws in non-ICs has involved very little or no understanding of the dynamic that operates in the economic and technological domain of non-ICs. 